\title{
31. GENERATION AND MIGRATION OF LOW-MOLECULAR-WEIGHT HYDROCARBONS IN SEDIMENTS OF DEEP SEA DRILLING PROJECT LEG 79, SITES 544, 545, AND 547, OFFSHORE MOROCCO'
}

\author{
Rainer G. Schaefer, Detlev Leythaeuser, and James Gormly, Institut für Erdöl und Organische Geochemie, Jülich ${ }^{2}$
}

\begin{abstract}
A series of core samples taken during Cruise 79 of Glomar Challenger, drilling offshore Morocco (Mazagan Plateau), is analyzed for their low-molecular-weight hydrocarbon contents. Fifty-four samples from DSDP Holes 544A, $545,547 \mathrm{~A}$, and 547B, deep frozen on board immediately after recovery, are studied by a hydrogen-stripping/thermovaporization technique combined with capillary gas chromatography. Thirty-eight compounds in the $\mathrm{C}_{2}-\mathrm{C}_{8}$ molecular range, including saturated, olefinic, and aromatic hydrocarbons, are identified.

Because of large differences in organic carbon contents, the total $\mathrm{C}_{2}-\mathrm{C}_{8}$ hydrocarbon concentrations vary from about 20 to $1500 \mathrm{ng} / \mathrm{g}$ dry sediment weight in the whole sample series. Organic-carbon normalized values are about $3.2 \times 10^{4} \mathrm{ng} / \mathrm{g} \mathrm{C}_{\text {org }}$ for Lithologic Subunits IIIA and IIIB at Site 545 (Cenomanian to Aptian) and $1.0 \times 10^{5} \mathrm{ng} / \mathrm{g} \mathrm{C}_{\text {org }}$ for Unit V at Site 547 (Cenomanian to Albian) reflecting the slightly more advanced maturity stage at the latter site. Values exceeding $10^{5} \mathrm{ng} / \mathrm{g} \mathrm{C}_{\text {org }}$ (Site 545) and $2 \times 10^{5} \mathrm{ng} / \mathrm{g} \mathrm{C}_{\text {org }}$ (Site 547) are associated with samples that are very lean in organic carbon and are generally rich in carbonate. These samples are enriched by small amounts of gaseous hydrocarbons.

A detailed study of individual hydrocarbon concentrations, plotted against depth, reveal additional indications for migration phenomena. At Site 547, for instance, the most mobile hydrocarbons studied (e.g., ethane) appear to migrate by diffusion or a related process from more than $700 \mathrm{~m}$ depth toward the surface.
\end{abstract}

\section{INTRODUCTION}

During the cruise of Glomar Challenger in the northeastern Atlantic offshore Morocco (DSDP Leg 79, April and May 1981), 54 samples (1 from Hole 544A, 25 from Site 545, 14 from Hole 547A, and 14 from Hole 547B) were collected for this study in sealed vials and deep frozen on board. Most of the core samples studied were found to be severely contaminated by acetone, which is routinely used as a solvent during sampling procedures on board Glomar Challenger.

It is commonly accepted that the quantity and distribution of low-molecular-weight hydrocarbons $\left(C_{1}-C_{8}\right.$ range) represent a sensitive indicator for the thermally controlled hydrocarbon generation processes in low-maturity sediments (see, e.g., Whelan et al., 1980; Whelan and Hunt, 1981, 1982; Schaefer, Leythaeuser, et al., 1983; Schaefer, von der Dick, et al., 1983; Schaefer and Leythaeuser, 1984, and references cited therein). The exception is methane which can, in addition, be formed in large quantities by bacterial carbonate reduction or acetic acid fermentation-type reactions. Recently, Hunt et al. (1980a) and Whelan et al. (1982) found traces of biogenic hydrocarbons (molecular range above $\mathrm{C}_{2}$ ) originating from marine bacteria and algae and, therefore, a dual origin of low-molecular-weight hydrocarbons in such sediments has to be assumed. However, it is not yet clear if this biogenic origin may account for a sizable proportion of the total $\mathrm{C}_{2}-\mathrm{C}_{8}$ hydrocarbon fraction.

\footnotetext{
${ }^{1}$ Hinz, K., Winterer, E. L., et al., Init. Repts. DSDP, 79: Washington (U.S. Govt. Printing Office).

2 Addresses: (Schaefer, Leythaeuser) Institut für Erdöl und Organische Geochemie, ICH-5,
Office) KFA-Jülich, D-5170 Jülich, Federal Republic of Germany; (Gormly, present address) Mobil Research and Development Corporation, Research Division, Farmers Branch, Texas 75234.
}

Owing to their high mobility in the water-saturated pore space, the study of the low-molecular-weight compounds also represents a valuable tool to recognize redistribution or migration processes in the subsurface (Whelan, 1979; Thompson, 1979; Leythaeuser et al., $1979,1983)$. It was, therefore, the objective of the present study to look for compositional features in the light hydrocarbon fraction that can be interpreted in this sense.

The study benefited from two extensive sample series collected at Sites 545 and 547, both comprising thick sequences of Cretaceous nannofossil-bearing claystones (Lithologic Subunits IIIA, IIIB, and Unit V, respectively) overlain by nannofossil ooze and chalk (Table 1, Figs. 1 and 10). Lithologic Subunit IIIC, sampled at Site 545, consists of nannofossil ooze and microdolosparite, whereas Subunit VIA of Site 547 is a conglomeratic limestone. For a detailed description of lithologic units and stratigraphy, see the site chapters (this volume).

\section{METHODS}

Fifty-four small sediment samples (approximately $10 \mathrm{~g}$ ) were collected on board immediately after the cores had been cut into sections. Samples were stored in polytetrafluoroethene foil sealed $20 \mathrm{ml}$ screwcapped aluminum containers in a deep freezer and remained deep frozen (approximately $-20^{\circ} \mathrm{C}$ ) until the analyses were performed in the laboratory at KFA-Jülich. The low-molecular-weight hydrocarbons (molecular range $\mathrm{C}_{2}-\mathrm{C}_{8}$ ) were analyzed by a slightly modified, combined hydrogen-stripping/thermovaporization method described in detail by Schaefer et al. (1978). The modification of the method was necessary because most of the core samples turned out to be extensively contaminated by acetone. Similar contamination effects have been documented in samples from various DSDP holes (Schaefer, von der Dick, et al., 1983, Schaefer and Leythaeuser, 1984). The severe contamination is caused by the usage of large quantities of acetone during on board core-cutting and sealing procedures, in particular, by rinsing the plastic caps with this solvent before they are pressed on the core-liner segments. Obviously, the acetone penetrates and contaminates the cores. 
In many samples the concentration of acetone by far exceeded that of the total light hydrocarbons.

The analytical method used in this study comprises both the extraction of hydrocarbons from the rock and subsequent capillary gas chromatography in a single-step procedure carried out in a closed gasflow system (Schaefer et al., 1978). Briefly, a small portion of the freshly crushed sediment sample (generally about $1 \mathrm{~g}$ ) is placed in the flow system of a capillary gas chromatograph, the hydrogen carrier gas serving as the stripping gas. Simultaneously the rock sample is heated to $110^{\circ} \mathrm{C}$ for $5 \mathrm{~min}$. in a hydrogen flow of $5 \mathrm{ml} / \mathrm{min}$. Prior to the chromatographic separation, the mobilized compounds are passed through a filter of a mixture of dry $\mathrm{CaCl}_{2}$ and silica gel in order to prevent the pore water and the acetone from entering the gas chromatographic column [for analytical details see Schaefer, von der Dick, et al. (1983) and Schaefer and Leythaeuser (1984)]. We have indications that the yields obtained by this procedure represent nearly the absolute quantities of light hydrocarbons present, both dissolved in the pore water and adsorbed on mineral surfaces. Hence, the concentration values obtained cannot be compared easily to other light hydrocarbon data (e.g., from headspace analysis).

Organic carbon contents of the sediments have been measured on the thermovaporized samples after treatment with hot $6 \mathrm{~N} \mathrm{HCl}$ by a combustion method (LECO Carbon Analyzer IR 112). The accuracy of this method is still rather high at organic carbon levels of $0.1 \%(10 \%$ relative standard deviation) or even less. Values near $0.01 \%$, however, although still above detection limit, are considered with care in the following discussion. Data on kerogen type were obtained by application of the Rock-Eval pyrolysis procedure (Espitalié et al., 1977).

\section{RESULTS AND DISCUSSION}

\section{Site 544}

Only one sample from Hole 544A (latitude $33^{\circ}$ $46.00^{\prime} \mathrm{N}$, longitude $9^{\circ} 24.26^{\prime} \mathrm{W}$ ) was analyzed. The slightly clayey nannofossil ooze from $57.5 \mathrm{~m}$ depth had only $0.11 \%$ organic carbon, a hydrogen index of $13 \mathrm{mg}$ hydrocarbons $/ \mathrm{g} \mathrm{C}_{\mathrm{org}}$, and, accordingly, a very low light hydrocarbon content $(21 \mathrm{ng} / \mathrm{g}$ dry sediment). See Table 1 for concentrations of individual compounds.

\section{Site 545}

Site 545 (latitude $33^{\circ} 39.86^{\prime} \mathrm{N}$, longitude $9^{\circ} 21.88^{\prime} \mathrm{W}$ ) was drilled near the foot of the steep part of the Mazagan Escarpment at a place where older Mesozoic strata are blanketed by only a thin wedge of Cenozoic slope sediments. Twenty-five samples covering the depth interval from $86 \mathrm{~m}$ (Core 545-10) to 530.5 m (Core 545-56) were analyzed for this study.

The organic carbon contents are fairly low, ranging from virtually 0 to $1 \%$ (Table 1 and Fig. 1). The higher values are found mainly in the Lower Cretaceous. The results of the Rock-Eval pyrolysis (Table 1) classify the organic matter throughout this hole as kerogen type III. The hydrogen indexes are below $175 \mathrm{mg}$ hydrocarbons $/ \mathrm{g}$ $\mathrm{C}_{\text {org }}$ and oxygen indexes frequently exceed $200 \mathrm{mg} \mathrm{CO}$ / $\mathrm{g} \mathrm{C}_{\text {org }}$, indicative of a strong terrestrial contribution. $\mathrm{T}_{\max }$ values are consistently below $425^{\circ} \mathrm{C}$, suggesting a very low level of maturity. These conclusions are in accordance with results on amount and type of organic matter at Site 545, including the comparison between on board and shore-based measurements, discussed in detail by Rullkötter et al. (this volume).

Low-molecular-weight hydrocarbon concentrations, normalized to dry sediment weight, are listed in Table 1. The total light hydrocarbon $\left(\mathrm{C}_{2}-\mathrm{C}_{8}\right)$ concentrations from Site 545 are plotted against depth in Figure 1, both in absolute and organic-carbon normalized units (ng/g of dry sediment weight and ng/g of organic carbon, respectively). It is apparent from Figure 1 that hydrocarbon concentrations vary over the whole depth range by more than one order of magnitude, that is, from about $30 \mathrm{ng}$ / $\mathrm{g}$ in the Miocene nannofossil chalk $(212.5 \mathrm{~m}$, Core $545-$ 23) to $550 \mathrm{ng} / \mathrm{g}$ in the nannofossil-bearing claystone of Albian age at $402.5 \mathrm{~m}$ depth (Core 545-43). The claystones and calcareous mudstones of late Aptian age (Cores $545-55$ and 545-56) contain 200 to $300 \mathrm{ng} / \mathrm{g}$. These values are, despite a somewhat lower maturity $\overline{(R}_{o}=0.3 \%$ in the late Aptian according to Rullkötter et al., this volume), much higher than in calcareous mudstone/claystone samples of DSDP Hole 416A (deep Moroccan Basin). Whelan and Hunt (1980) measured $\mathrm{C}_{2}-\mathrm{C}_{7}$ hydrocarbon concentrations of $42.6 \mathrm{ng} / \mathrm{g}\left(6980 \mathrm{ng} / \mathrm{g} \mathrm{C}_{\text {org }}\right)$ and $54.1 \mathrm{ng} / \mathrm{g}\left(12,300 \mathrm{ng} / \mathrm{g} \mathrm{C}_{\text {org }}\right)$ in two Valanginian age samples of comparable kerogen type and organic carbon contents of 0.61 and $0.44 \%$, respectively, at a maturity-level equivalent to a vitrinite reflectance of about $0.4 \%$ (Boutefeu et al., 1980). Their measurements were made, however, with a different analytical method comprising a slightly different molecular range.

In line with their very low organic carbon contents, the three lowermost samples studied at Site 545 are very lean in light hydrocarbons $(40-50 \mathrm{ng} / \mathrm{g}$ ). To eliminate the influence of fluctuations in organic carbon contents, the hydrocarbon concentrations also are shown in Figure 1 in organic-carbon-normalized units. There is, however, an even greater total variation with depth than in the absolute concentration values. This is partly due to the reduced accuracy of organic carbon determinations at low organic carbon levels. Hydrocarbon concentrations vary from $6 \times 10^{3}$ to $5 \times 10^{5} \mathrm{ng} / \mathrm{g} \mathrm{C}_{\text {org }}$. Obviously, the highest values are associated with organic carbon lean samples $\left(\mathrm{C}_{\text {org }}<0.1 \%\right)$. It is suspected, therefore, that they are enriched by small amounts of migrated light hydrocarbon compounds. All other samples analyzed $\left(C_{\text {org }} \geq 0.1 \%\right)$, except for two samples of Lithologic Unit II, do not reveal much variation of their organic-carbon-normalized values on a logarithmic scale over the entire depth interval. Mean concentrations for these 19 samples is $3.2 \times 10^{4} \mathrm{ng} / \mathrm{g} \mathrm{C}_{\text {org }}$. Values of this order of magnitude have frequently been observed for Type III kerogen-bearing sediments that are in an immature evolution stage [cf., e.g., our reports about DSDP Leg 71 and 75 sediments (Schaefer, von der Dick, et al., 1983; Schaefer and Leythaeuser, 1984, respectively)].

The absolute concentrations of the individual $n$-alkanes-ethane, propane, butane, pentane, hexane, and heptane-as well as toluene are shown in Figures 2 and 3 . On the whole for all these compounds, the depthtrends appear to vary in phase, showing highest values between 320 and $400 \mathrm{~m}$, and around $535 \mathrm{~m}$ depth. The variation in concentration over the entire depth interval is, however, much more pronounced for the higher molecular weight compounds than for ethane and propane. Whereas ethane concentrations change only by a factor of five, $n$-heptane varies by nearly two orders of magnitude between the nannofossil chalk $(212.5 \mathrm{~m}, 0.95 \mathrm{ng} / \mathrm{g})$ and the nannofossil claystone $(523 \mathrm{~m}, 68 \mathrm{ng} / \mathrm{g})$. We interpret these differences as an indication for a redistrib- 
ution process that has preferentially affected the gaseous compounds.

As shown in Figures 4 and 5 the corresponding organic-carbon-normalized concentrations are relatively constant throughout Lithologic Unit III except for the nannofossil ooze $(527.5 \mathrm{~m})$ and the microdolosparite (530.5 $\mathrm{m})$, which have higher concentrations. Similarly, high values are encountered in Lithologic Subunit IB, whereas in Unit II the concentrations are much lower than in most of Unit III. The drastic change in light hydrocarbon concentrations between Unit II (Core 545-27) and Subunit IIIA (Core 545-29) coincides well with the change in lithology from chalk to claystone at the Neogene/mid-Cretaceous unconformity at $253 \mathrm{~m}$ depth. A comparison of, for instance, the lowermost sample of the chalk (Sample 545-27-1, 90-93 cm) with the uppermost sample of the claystone interval (Sample 545-29-1, $20-22 \mathrm{~cm}$ ) that have very similar organic carbon contents and broadly comparable pyrolysis yields (see Table 1) leads to the conclusion that most of the chalk interval is probably depleted in light hydrocarbons. This effect is most pronounced for $n$-pentane, $n$-hexane, and $n$-heptane, whereas it is least pronounced for the gases.

This difference between the gases and their higher molecular weight homologs can be explained by the assumption of a lateral water flow, removing hydrocarbons from this section and a subsequent replenishment of the depleted interval by migrated gases. The transport mechanism for this replenishment could be diffusion which favors the gaseous compounds due to their high diffusivities. The strong relative enrichment of the same interval by gaseous compounds is also obvious from Figure 6. Both the relative $\mathrm{C}_{2}-\mathrm{C}_{5}$ content (as a proportion of the total light hydrocarbon fraction) and the ethane $/ n$-heptane ratio reveal a significant increase between Lithologic Units III and II and also for the lowermost three samples of Unit III. In particular, the ethane $/ n$-heptane ratio increases by a factor of 10 from the center part of Unit III to Subunit IB (Sample 545$19-5,13-15 \mathrm{~cm}$ ).

A similar phenomenon, that is, a selective enrichment by a particular compound, is shown in Figure 7. The toluene $/ \mathrm{C}_{7}$-cycloalkane ratio remains fairly low from the Neogene/mid-Cretaceous unconformity down to about $520 \mathrm{~m}$. Markedly higher ratios occur again in Units I, II, and the lowermost part of Unit III. Similarly high toluene/methylcyclohexane ratios in sediments of DSDP Site 511, Leg 71, were interpreted as indications of preferential adsorption of toluene on the mineral surfaces from hydrocarbon-bearing formation waters that have moved laterally updip (Schaefer, Leythaeuser, et al., 1983). We interpret the data shown in Figure 7 in the same sense. However, at Site 545 these anomalies also are associated in part with significant lithologic changes, for example, from nannofossil claystone and calcareous mudstone (Subunit IIIB) to nannofossil ooze and microdolosporite (Subunit IIIC).

Remarkably high values in the methylpropane/ $n$-butane and methylbutane/ $n$-pentane ratios (Fig. 8) are observed in the upper part of Lithologic Unit III, whereas they remain relatively constant in all other samples $(0.5-$
0.7 for methylpropane/ $n$-butane, and $0.4-0.8$ for methylbutane/ $n$-pentane). The strong relative increase of the $\mathrm{C}_{5}$-isoalkanes compared to $n$-pentane from about 300 to $400 \mathrm{~m}$ depth also is seen in Figure 9 where the composition of the $\mathrm{C}_{5}$-hydrocarbons (sum of $n$-pentane, branched alkanes, and cyclopentane concentrations normalized to $100 \%$ ) is plotted against depth. It is not clear currently whether the very high isoalkane/ $n$-alkane ratios in the upper part of Unit III are the result of a redistribution process by diffusion (the branched isomer has a slightly higher diffusivity if compared to the normal alkane) or are due to differences in the source type. However, it is unlikely that this phenomenon is source controlled since even samples of similar kerogen type, maturity, and lithology (e.g., samples from 323.5 and $513.3 \mathrm{~m}$ depth, both characterized as bioturbated nannofossil claystones) reveal strong differences in their isoalkane $/ n$-alkane ratios. The relative content of cyclopentane (Fig. 9 , left) is in most samples between 2 and $4 \%$ (mean value $3.0 \%$ ), which is typical for immature sediments.

Strong relative enrichment in toluene, compared to the remaining $C_{7}$ hydrocarbons in the lowermost part of Unit III and in Units I and II, is also shown in Figure 9 (right). This, again, as in Figure 7, may be at least partly due to the preferential adsorption of the most polar compounds on the mineral surfaces from the lateral updip migration of hydrocarbon-bearing formation waters. However, no conclusion can be reached at present as to the role that specific minerals play in this adsorption process. The two samples from Subunit IIIB at $524.5 \mathrm{~m}$ depth (nannofossil-bearing calcareous mudstone) and $526.0 \mathrm{~m}$ depth (nannofossil-rich calcareous claystone), for instance, have extremely different relative toluene contents.

\section{Site 547}

Site 547 (latitude $33^{\circ} 46.84^{\prime} \mathrm{N}$, longitude $9^{\circ} 20.98^{\prime} \mathrm{W}$ ) is located on the seaward (NE) margin of the Site 544 structural high at a place where acoustic basement is covered by more than $1000 \mathrm{~m}$ of pelagic sediments. Fourteen samples each from Hole 547A (Cores 547A-5 to 547A-71) and from Hole 547B (Cores 547B-4 to 547B-6), covering the depth interval $114-777 \mathrm{~m}$, were analyzed.

The level of organic carbon contents (Table 1 and Fig. 10) in the Cenomanian and younger sediments is low and ranges between 0.01 and about $1 \%$. In the Albian the organic carbon contents range primarily between 1 and $2 \%$, with a high value of $2.3 \%$. The Jurassic samples contain essentially no organic carbon. The RockEval pyrolysis data (Table 1) indicate that most of the samples analyzed are type III kerogens with hydrogen index values below $140 \mathrm{mg}$ hydrocarbons/g $\mathrm{C}_{\mathrm{org}}$ and many oxygen index values in excess of $200 \mathrm{mg} \mathrm{CO} / \mathrm{g}$ $\mathrm{C}_{\text {org. }}$. There are two exceptions of samples bearing an improved kerogen quality-at $771.7 \mathrm{~m}$ in the lower part of the Albian where a hydrogen index of $620 \mathrm{mg}$ hydrocarbons $/ \mathrm{g} \mathrm{C}_{\text {org }}$ indicates a type II kerogen. The other exception is an Eocene-age sample at $252 \mathrm{~m}$ depth that bears a mixed Type II-III $\left(I_{H}=220 \mathrm{mg}\right.$ hydrocarbons $/ \mathrm{g}$ $\left.\mathrm{C}_{\text {org }}, \bar{I}_{O}=167 \mathrm{mg} \mathrm{CO} / \mathrm{g} \mathrm{C}_{\text {org }}\right)$. With $T_{\max }$ values less than $430^{\circ} \mathrm{C}$, all samples in these holes would be classi- 
Table 1. Summary of low-molecular-weight hydrocarbon concentrations, organic carbon contents, Rock-Eval pyrolysis data, and lithology of core samples from DSDP Holes 544A, 545, 547A, and 547B, Leg 79 (lithologic description as defined by the shipboard scientists).

\begin{tabular}{|c|c|c|c|c|c|c|}
\hline & $\begin{array}{l}\text { Sample (interval in cm) } \\
\text { Depth (m) }\end{array}$ & $\begin{array}{l}544 \mathrm{~A}-7-3,0-3 \\
57.5\end{array}$ & $\begin{array}{c}545-10-1,148-150 \\
86.0\end{array}$ & $\begin{array}{c}545-14-4,40-42 \\
127.4\end{array}$ & $\begin{array}{c}545-19-5,13-15 \\
176.1\end{array}$ & $\begin{array}{l}545-23-4,0-2 \\
212.5\end{array}$ \\
\hline & Lithology & $\begin{array}{c}\text { Slightly } \\
\text { clayey } \\
\text { nannofossil } \\
\text { ooze }\end{array}$ & $\begin{array}{c}\text { Clayey } \\
\text { foraminiferal } \\
\text { nannofossil } \\
\text { ooze }\end{array}$ & $\begin{array}{c}\text { Slightly } \\
\text { clayey } \\
\text { foraminiferal } \\
\text { nannofossil } \\
\text { ooze }\end{array}$ & $\begin{array}{c}\text { Slightly } \\
\text { clayey } \\
\text { nannofossil } \\
\text { ooze, } \\
\text { burrows }\end{array}$ & $\begin{array}{c}\text { Foraminiferal- } \\
\text { nannofossil } \\
\text { chalk, } \\
\text { burrows }\end{array}$ \\
\hline & $\mathrm{C}_{\text {org }}[\%$ dry sediment weight] & 0.11 & 0.19 & 0.01 & 0.02 & 0.29 \\
\hline & Hydrogen index [mg hydrocarbons/g C & 13 & 52 & $\mathrm{i}$ & $\mathrm{i}$ & 12 \\
\hline & Oxygen index $\left[\mathrm{mg} \mathrm{CO}_{2} / \mathrm{g} \mathrm{C}_{\mathrm{org}}\right]$ & 186 & 438 & $\mathrm{i}$ & $\mathrm{i}$ & 214 \\
\hline & $T_{\max }\left[{ }^{\circ} \mathrm{C}\right]$ & $398 / 439 / 532$ & $412 / 527$ & 525 & 412 & 409 \\
\hline Ref. No. & Hydrocarbon & \multicolumn{5}{|c|}{ Concentration ( $\mathrm{ng} / \mathrm{g}$ dry sediment weight) } \\
\hline 1 & Ethene & 0.80 & 0.80 & 0.42 & 1.7 & 1.4 \\
\hline 2 & Ethane & 2.9 & 7.3 & 5.6 & 4.5 & 3.4 \\
\hline 3 & Propene & 1.3 & 2.2 & 1.5 & 2.0 & 2.0 \\
\hline 4 & Propane & 3.7 & 9.5 & 8.5 & 6.4 & 4.9 \\
\hline 5 & Methylpropane & 1.7 & 3.2 & 3.3 & 2.5 & 2.1 \\
\hline 6 & Methylpropene + 1-butene & 1.4 & 2.3 & 2.1 & 2.7 & 2.8 \\
\hline 7 & n-Butane & 2.3 & 6.3 & 4.6 & 3.2 & 2.7 \\
\hline 8 & trans-2-Butene & 0.28 & 0.56 & 0.42 & 0.68 & 0.52 \\
\hline 9 & 2,2-Dimethylpropane & 0.08 & 0.11 & 0.06 & 0.07 & 0.12 \\
\hline 10 & cis-2-Butene & 0.20 & 0.44 & 0.37 & 0.52 & 0.35 \\
\hline 11 & Methylbutane & 0.82 & 1.9 & 1.6 & 1.3 & 1.1 \\
\hline 12 & $n$-Pentane & 1.9 & 5.1 & 3.0 & 2.3 & 2.0 \\
\hline 13 & 2,2-Dimethylbutane & $<0.01$ & $<0.01$ & $<0.01$ & $<0.01$ & $<0.01$ \\
\hline 14 & Cyclopentane & $<0.1$ & 0.23 & $<0.1$ & 0.12 & $<0.1$ \\
\hline 15 & 2,3-Dimethylbutane & $<0.1$ & - & 0.15 & $<0.1$ & 0.82 \\
\hline 16 & 2-Methylpentane & 0.31 & 0.74 & 0.65 & 0.42 & 0.99 \\
\hline 17 & 3-Methylpentane & 0.29 & 0.66 & 0.47 & 0.47 & 0.35 \\
\hline 18 & $n$-Hexane & 1.4 & 4.1 & 2.1 & 1.7 & 1.7 \\
\hline 19 & Methylcyclopentane + 2,2-dimethylpentane & 0.12 & 0.40 & 0.23 & 0.26 & $<0.1$ \\
\hline 20 & 2,4-Dimethylpentane & $<0.1$ & $<0.1$ & $<0.1$ & $<0.1$ & $<0.1$ \\
\hline 21 & Benzene & 1.2 & 3.2 & 1.0 & 1.4 & 1.6 \\
\hline 22 & Cyclohexane & $<0.1$ & $<0.1$ & $<0.1$ & $<0.1$ & 0.12 \\
\hline 23 & 2-Methylhexane & 0.16 & 0.40 & 0.19 & 0.11 & 0.11 \\
\hline 24 & 2,3-Dimethylpentane + 1,1-dimethylcyclopentane & $<0.1$ & $<0.1$ & 0.12 & $<0.1$ & $<0.1$ \\
\hline 25 & 3-Methylhexane & 0.16 & 0.39 & 0.18 & 0.11 & 0.12 \\
\hline 26 & 1,cis-3-Dimethylcyclopentane & 0.16 & 0.39 & 0.18 & 0.33 & 0.12 \\
\hline 27 & 1,trans-3-Dimethylcyclopentane & $<0.1$ & $<0.1$ & $<0.1$ & $<0.1$ & $<0.1$ \\
\hline 28 & 1,trans-2-Dimethylcyclopentane & $<0.1$ & $<0.1$ & $<0.1$ & $<0.1$ & $<0.1$ \\
\hline 29 & $n$-Heptane & 1.3 & 3.1 & 1.4 & 1.3 & 0.95 \\
\hline 30 & 1,cis-2-Dimethylcyclopentane & $<0.1$ & $<0.1$ & $<0.1$ & $<0.1$ & $<0.1$ \\
\hline 31 & Methylcyclohexane & $<0.1$ & 0.19 & 0.10 & $<0.1$ & $<0.1$ \\
\hline 32 & 2,5-Dimethylhexane & - & - & - & - & $<0.1$ \\
\hline 33 & Ethylcyclopentane & $<0.1$ & 0.15 & $<0.1$ & $<0.1$ & $<0.1$ \\
\hline 34 & 1 ,trans-2,cis-4-Trimethylcyclopentane & $<0.1$ & $<0.1$ & $<0.1$ & $<0.1$ & - \\
\hline 35 & 1 ,trans-2,cis-3-Trimethylcyclopentane & $<0.1$ & $<0.1$ & $<0.1$ & $<0.1$ & - \\
\hline 36 & Toluene & 1.5 & 5.7 & 5.0 & 3.2 & 3.2 \\
\hline 37 & 2-Methylheptane & $<0.1$ & 0.36 & 0.16 & $<0.1$ & - \\
\hline 38 & $n$-Octane & 1.1 & 2.9 & 1.7 & 0.88 & 0.95 \\
\hline
\end{tabular}

Note: $\mathrm{i}=$ value insignificant (pyrolysis data); $-=$ concentration below or near detection limit (light hydrocarbons).

fied as immature. Organic carbon contents and pyrolysis yields of Site 547 sediments, including the comparison between on-board and shore-based measurements, are discussed in more detail by Rullkötter et al. (this volume).

Low-molecular-weight hydrocarbon concentrations, normalized to dry sediment weight, are listed in Table 1. The total light hydrocarbon $\left(\mathrm{C}_{2}-\mathrm{C}_{8}\right)$ concentrations for Holes 547A and 547B are plotted against depth in Figure 10, both in rock-weight-based and organic-carbon normalized units. As at Site 545, hydrocarbon concentrations at Site 547 vary considerably over the whole depth range, that is, by nearly two orders of magnitude. Lowest values are found in the very organic carbon lean samples of Jurassic age (20-50 ng/g of rock) whereas values of about $1000 \mathrm{ng} / \mathrm{g}$ are measured in the lower Cenomanian and most of the Albian section. A gradual decrease in total light hydrocarbons is observed from $772 \mathrm{~m}$ upward.

Fluctuations in the organic-carbon-normalized values (Fig. 10) are considerable, in particular in Lithologic Units I to Subunits IVB and VIA. The samples of Unit I and Subunits IIB, IVA (Sample 547A-30-3, 0-2 cm), and VIA reveal values exceeding $2 \times 10^{5} \mathrm{ng} / \mathrm{g} \mathrm{C}_{\text {org }}$ and, therefore, appear to be enriched by migrated hydrocarbons. However, because of their very low organic carbon contents and due to the reduced accuracy at these levels, the organic-carbon-normalized values are extremely sensitive to even the slightest hydrocarbon impregnation that in samples of higher organic-carbon contents would not be significant. Most samples of the Cenomanian and Albian, on the other hand, are close to the average of $1.0 \times 10^{5} \mathrm{ng} / \mathrm{g} \mathrm{C}_{\text {org }}$ for 16 samples from Unit V. Comparing this value with Lithologic Subunits IIIA and IIIB 
Table 1. (Continued).

\begin{tabular}{|c|c|c|c|c|c|c|c|c|}
\hline $\begin{array}{c}545-27-1,90-93 \\
246.9\end{array}$ & $\begin{array}{c}545-29-1,20-22 \\
265.2\end{array}$ & $\begin{array}{c}545-35-2,0-2 \\
323.5\end{array}$ & $\begin{array}{c}545-39-2,0-2 \\
361.5\end{array}$ & $\begin{array}{c}545-43-4,0-1 \\
402.5\end{array}$ & $\begin{array}{c}545-47-2,48-50 \\
438.0\end{array}$ & $\begin{array}{c}545-47-3,117-119 \\
440.2\end{array}$ & $\begin{array}{c}545-51-3,0-2 \\
477.0\end{array}$ & $\begin{array}{c}545-55-1,133-135 \\
513.3\end{array}$ \\
\hline $\begin{array}{l}\text { Clayey } \\
\text { nannofossil } \\
\text { chalk }\end{array}$ & $\begin{array}{r}\text { Nanno } \\
\text { clayst } \\
\text { bioturb }\end{array}$ & $\begin{array}{l}\text { ossil } \\
\text { one } \\
\text { ated }\end{array}$ & $\begin{array}{l}\text { Nannofossil- } \\
\text { bearing } \\
\text { claystone } \\
\text { bioturbated }\end{array}$ & $\begin{array}{l}\text { Nannofossil- } \\
\text { bearing } \\
\text { claystone } \\
\text { burrows }\end{array}$ & \multicolumn{4}{|c|}{$\begin{array}{c}\text { Nannofossil- } \\
\text { bearing } \\
\text { claystone } \\
\text { bioturbated }\end{array}$} \\
\hline 0.57 & 0.47 & 0.62 & 1.00 & 1.01 & 0.42 & 0.89 & 0.31 & 0.38 \\
\hline 109 & 32 & 59 & 123 & 132 & 75 & 134 & 56 & 61 \\
\hline 214 & 203 & 222 & 172 & 124 & 271 & 147 & 229 & 236 \\
\hline 416 & 416 & 412 & 419 & 423 & 418 & 423 & 422 & 418 \\
\hline \multicolumn{9}{|c|}{ Concentration (ng/g dry sediment weight) } \\
\hline 2.0 & 3.2 & 1.5 & 2.9 & 1.9 & 1.4 & 1.4 & 0.99 & 1.1 \\
\hline 4.6 & 8.9 & 10.2 & 10.4 & 10.7 & 3.9 & 10.9 & 4.1 & 3.0 \\
\hline 2.1 & 5.4 & 2.1 & 3.4 & 2.0 & 1.7 & 1.4 & 1.2 & 0.81 \\
\hline 6.1 & 12.7 & 16.2 & 22.2 & 25.6 & 6.6 & 24.4 & 6.7 & 4.8 \\
\hline 3.8 & 8.6 & 28.9 & 23.8 & 30.4 & 4.7 & 14.7 & 2.7 & 2.1 \\
\hline 2.1 & 5.1 & 2.4 & 4.2 & 1.8 & 0.86 & 1.7 & 1.4 & 0.94 \\
\hline 3.2 & 8.5 & 18.5 & 26.2 & 23.7 & 5.9 & 17.1 & 4.6 & 3.4 \\
\hline 0.79 & 1.8 & 0.77 & 1.2 & 1.1 & 1.0 & 1.0 & 0.74 & 0.34 \\
\hline 0.19 & 0.26 & 0.52 & 0.62 & 0.92 & 0.34 & 0.64 & 0.21 & 0.12 \\
\hline 0.47 & 1.3 & 0.50 & 0.95 & 0.59 & 0.43 & 0.47 & 0.29 & 0.23 \\
\hline 1.6 & 10.4 & 36.9 & 51.7 & 66.7 & 14.6 & 46.1 & 6.7 & 4.3 \\
\hline 2.5 & 11.1 & 30.4 & 41.9 & 44.0 & 11.8 & 32.1 & 7.7 & 5.5 \\
\hline$<0.01$ & 0.17 & $<0.01$ & 0.61 & 0.97 & 1.7 & 0.87 & $<0.01$ & 0.13 \\
\hline$<0.1$ & 0.55 & 2.2 & 3.9 & 10.0 & 0.86 & 2.5 & 0.38 & 0.26 \\
\hline$<0.1$ & 0.52 & 1.3 & 1.6 . & 2.1 & 0.60 & 1.5 & 0.30 & 0.26 \\
\hline 0.48 & 8.3 & 25.1 & 34.2 & 42.8 & 13.0 & 32.9 & 8.1 & 6.2 \\
\hline 0.41 & 2.0 & 3.6 & 5.3 & 6.1 & 2.2 & 5.0 & 1.3 & 0.83 \\
\hline 1.5 & 12.5 & 23.8 & 35.0 & 48.0 & 15.6 & 39.9 & 11.6 & 8.7 \\
\hline 0.20 & 2.5 & 12.7 & 12.8 & 10.4 & 2.3 & 5.7 & 1.3 & 0.78 \\
\hline$<0.1$ & 0.59 & 0.76 & 0.74 & 1.6 & 0.39 & 0.94 & 0.24 & 0.17 \\
\hline 2.2 & 4.0 & 9.3 & 11.0 & 10.3 & 3.1 & 7.3 & 2.7 & 1.6 \\
\hline 0.12 & 0.64 & 2.3 & 3.6 & 4.9 & 1.4 & 3.5 & 0.76 & 0.43 \\
\hline 0.13 & 1.9 & 2.9 & 4.1 & 7.9 & 2.6 & 6.0 & 1.8 & 1.3 \\
\hline 0.11 & 3.5 & 5.3 & 5.9 & 11.7 & 3.1 & 7.6 & 2.3 & 2.0 \\
\hline 0.13 & 1.7 & 2.6 & 3.4 & 7.4 & 2.2 & 5.1 & 1.5 & 1.1 \\
\hline 0.13 & 3.0 & 6.4 & 5.7 & 7.5 & 1.3 & 3.2 & 0.62 & 0.27 \\
\hline$<0.1$ & 2.2 & 7.1 & 6.2 & 7.6 & 1.0 & 3.0 & 0.38 & 0.20 \\
\hline 0.13 & 1.8 & 4.6 & 4.5 & 6.3 & 1.1 & 3.0 & 0.59 & 0.38 \\
\hline 2.6 & 16.1 & 19.9 & 33.6 & 61.8 & 15.9 & 44.1 & 15.3 & 13.0 \\
\hline$<0.1$ & 0.37 & 1.1 & 1.1 & 1.5 & $<0.1$ & 0.68 & 0.20 & 0.14 \\
\hline 0.26 & 2.7 & 6.6 & 7.5 & 13.6 & 3.6 & 8.8 & 2.4 & 1.3 \\
\hline- & $<0.1$ & 0.23 & 0.50 & 0.89 & 0.27 & 0.48 & 0.20 & 0.14 \\
\hline$<0.1$ & 1.1 & 2.2 & 2.7 & 4.4 & 0.87 & 2.6 & 0.78 & 0.41 \\
\hline- & 0.98 & 1.3 & 1.0 & 2.0 & 0.35 & 0.49 & 0.16 & 0.14 \\
\hline- & 0.97 & 1.4 & 2.7 & 3.3 & 0.80 & 2.2 & 0.65 & 0.41 \\
\hline 3.2 & 8.5 & 17.8 & 39.8 & 32.5 & 7.9 & 13.0 & 7.8 & 4.8 \\
\hline 0.21 & 4.8 & 6.7 & 12.6 & 28.3 & 6.0 & 14.8 & 5.6 & 4.6 \\
\hline 0.13 & 12.1 & 12.6 & 26.1 & 45.8 & 9.7 & 28.1 & 12.0 & 11.4 \\
\hline
\end{tabular}

at Site $545\left(3.2 \times 10^{4} \mathrm{ng} / \mathrm{g} \mathrm{C} \mathrm{C}_{\text {org }}\right)$, the conclusion appears warranted that at Site 547 the maturation as measured by these levels of light hydrocarbon generation is more advanced. This is also confirmed by vitrinite reflectance measurements of $\bar{R}_{o}=0.3 \%$ for the Aptian at Site 545 versus $\bar{R}_{o}=0.5 \%$ for the Albian at Site 547 (Rullkötter et al., this volume). The significantly higher maturity at Site 547 might be due to a higher heat flow caused by the proximity of a salt diapir that is located only about $6 \mathrm{~km}$ away from this site. The light hydrocarbon concentrations at Site 547 are, however, much lower than those of a mature source rock. Even the type II kerogen-bearing sample at $771.7 \mathrm{~m}$ depth (Sample 547B-5-7, 20-22 cm) only has a light hydrocarbon content of $83,000 \mathrm{ng} / \mathrm{g} \mathrm{C}_{\text {org. }}$. Similar values have been found for various low-mature "black shales" of DSDP Leg 71, Site 511 (Schaefer, von der Dick, et al., 1983) and Leg 75, Hole 530A (Schaefer and Leythaeuser, 1984). Light hydrocarbon concentrations in type II kerogen-bearing source beds in the main phase of petroleum generation $\left(\bar{R}_{o}=0.8-0.9 \%\right)$ are known to be about two orders of magnitude higher (Schaefer, Leythaeuser, et al., 1983).

For selected compounds, concentrations are plotted against depth on a dry sediment weight basis (Figs. 11 and 12) and in organic-carbon-normalized units (Figs. 13 and 14), respectively. Whereas the latter data reveal similar trends as the total $\mathrm{C}_{2}-\mathrm{C}_{8}$ fraction (Fig. 10), the rock-weight-based data reveal depth trends, the relative differences of which are controlled in a fairly regular fashion by the molecular weight of the compounds considered. This observation is reemphasized in Figure 15 where the $n$-alkane concentrations for Hole 547A (in ng/ $\mathrm{g}$ of rock, uniform logarithmic scale) are plotted versus depth. The variation with depth over the total interval is lowest for ethane and increases progressively with increasing carbon number. This relationship is just the opposite of what would be expected to result from relative differences in hydrocarbon-generation rates for this mo- 
Table 1. (Continued).

\begin{tabular}{|c|c|c|c|c|c|c|}
\hline & $\begin{array}{l}\text { Sample (interval in cm) } \\
\text { Depth (m) }\end{array}$ & $\begin{array}{c}545-55-2,81-83 \\
514.3\end{array}$ & $\begin{array}{l}545-55-3,96-98 \\
516.0\end{array}$ & $\begin{array}{l}545-55-4,102-104 \\
517.5\end{array}$ & $\begin{array}{l}545-55-5,92-94 \\
518.9\end{array}$ & $\begin{array}{l}545-55, \mathrm{CC}(17-19) \\
519.7\end{array}$ \\
\hline & Lithology & $\begin{array}{l}\text { Nannofossil- } \\
\text { bearing } \\
\text { claystone } \\
\text { bioturbated }\end{array}$ & $\begin{array}{l}\text { Nannofossil- } \\
\text { bearing } \\
\text { claystone }\end{array}$ & \multicolumn{2}{|c|}{$\begin{array}{l}\text { Nannofossil- } \\
\text { bearing } \\
\text { claystone } \\
\text { bioturbated }\end{array}$} & $\begin{array}{c}\text { Nannofossil- } \\
\text { bearing } \\
\text { claystone }\end{array}$ \\
\hline & $\mathrm{C}_{\text {org }}[\%$ dry sediment weight $]$ & 0.31 & 0.69 & 0.21 & 0.41 & 0.80 \\
\hline & Hydrogen index [mg hydrocarbons $/ \mathrm{g} \mathrm{C}_{\mathrm{org}}$ ] & 36 & 96 & 88 & 103 & 149 \\
\hline & Oxygen index $\left[\mathrm{mg} \mathrm{CO}_{2} / \mathrm{g} \mathrm{C}_{\mathrm{org}}\right]$ & 225 & 150 & 488 & 227 & 96 \\
\hline & $T_{\max }\left[{ }^{\circ} \mathrm{C}\right]$ & & 426 & 414 & 416 & 420 \\
\hline Ref. No. & Hydrocarbon & \multicolumn{5}{|c|}{ Concentration (ng/g dry sediment weight) } \\
\hline 1 & Ethene & 0.87 & 0.89 & 1.2 & 1.6 & 3.2 \\
\hline 2 & Ethane & 2.3 & 3.0 & 5.1 & 5.1 & 6.5 \\
\hline 3 & Propene & 1.1 & 0.87 & 1.5 & 1.9 & 4.0 \\
\hline 4 & Propane & 4.0 & 5.5 & 7.6 & 7.3 & 9.0 \\
\hline 5 & Methylpropane & 1.7 & 3.1 & 3.1 & 3.2 & 4.0 \\
\hline 6 & Methylpropene + 1-butene & 1.5 & 1.2 & 1.4 & 1.8 & 3.8 \\
\hline 7 & $n$-Butane & 2.3 & 3.9 & 4.8 & 4.6 & 6.2 \\
\hline 8 & trans-2-Butene & 0.32 & 0.46 & 0.45 & 0.60 & 1.3 \\
\hline 9 & 2,2-Dimethylpropane & 0.06 & 0.17 & 0.10 & 0.12 & 0.20 \\
\hline 10 & cis-2-Butene & 0.22 & 0.27 & 0.34 & 0.48 & 1.1 \\
\hline 11 & Methylbutane & 1.9 & 6.5 & 3.5 & 5.2 & 10.2 \\
\hline 12 & $n$-Pentane & 2.8 & 7.1 & 5.3 & 6.1 & 12.7 \\
\hline 13 & 2,2-Dimethylbutane & 0.05 & 0.19 & 0.10 & 0.14 & 0.36 \\
\hline 14 & Cyclopentane & 0.10 & 0.36 & 0.22 & 0.29 & 0.66 \\
\hline 15 & 2,3-Dimethylbutane & 0.12 & 0.47 & 0.21 & 0.34 & 0.95 \\
\hline 16 & 2-Methylpentane & 2.8 & 10.6 & 4.6 & 7.8 & 22.2 \\
\hline 17 & 3-Methylpentane & 0.47 & 1.2 & 0.78 & 1.2 & 3.1 \\
\hline 18 & $n$-Hexane & 4.3 & 13.3 & 6.7 & 9.5 & 28.1 \\
\hline 19 & Methylcyclopentane $+2,2$-dimethylpentane & 0.32 & 1.1 & 0.59 & 0.80 & 2.4 \\
\hline 20 & 2,4-Dimethylpentane & $<0.1$ & 0.27 & 0.11 & 0.19 & 0.62 \\
\hline 21 & Benzene & 1.4 & 1.9 & 1.5 & 2.2 & 4.1 \\
\hline 22 & Cyclohexane & 0.18 & 0.64 & 0.25 & 0.43 & 1.4 \\
\hline 23 & 2-Methylhexane & 0.60 & 2.2 & 1.0 & 1.6 & 4.8 \\
\hline 24 & 2,3-Dimethylpentane + 1,1-dimethylcyclopentane & 0.98 & 3.8 & 1.6 & 3.1 & 8.9 \\
\hline 25 & 3-Methylhexane & 0.50 & 1.8 & 0.82 & 1.3 & 4.1 \\
\hline 26 & 1,cis-3-Dimethylcyclopentane & 0.12 & 0.37 & 0.25 & 0.36 & 0.92 \\
\hline 27 & 1,trans-3-Dimethylcyclopentane & $<0.1$ & 0.29 & 0.13 & 0.20 & 0.65 \\
\hline 28 & 1,trans-2-Dimethylcyclopentane & 0.15 & 0.61 & 0.26 & 0.42 & 1.2 \\
\hline 29 & $n$-Heptane & 6.7 & 24.2 & 10.3 & 17.2 & 51.8 \\
\hline 30 & 1,cis-2-Dimethylcyclopentane & - & 0.18 & $<0.1$ & 0.11 & 0.39 \\
\hline 31 & Methylcyclohexane & 0.62 & 2.14 & 0.77 & 1.4 & 3.9 \\
\hline 32 & 2,5-Dimethylhexane & $<0.1$ & 0.18 & $<0.1$ & 0.11 & 0.39 \\
\hline 33 & Ethylcyclopentane & 0.18 & 0.87 & 0.45 & 0.70 & 1.9 \\
\hline 34 & 1,trans-2,cis-4-Trimethylcyclopentane & $<0.1$ & 0.27 & 0.10 & 0.21 & 0.68 \\
\hline 35 & 1,trans-2,cis-3-Trimethylcyclopentane & 0.18 & 0.75 & 0.26 & 0.56 & 1.1 \\
\hline 36 & Toluene & 5.0 & 4.9 & 7.0 & 3.3 & 19.1 \\
\hline 37 & 2-Methylheptane & 2.3 & 8.6 & 3.5 & 6.4 & 20.8 \\
\hline 38 & $n$-Octane & 5.4 & 18.6 & 8.6 & 13.0 & 39.6 \\
\hline
\end{tabular}

lecular range. In this case the gradient should become steeper with increasing carbon number (Tissot et al., 1971). The data of Tissot et al., however, were obtained for Toarcian-age shales of the Paris Basin representing a different type of organic material.

Nevertheless, we believe that the concentration depth patterns shown in Figure 15 result from hydrocarbon redistribution processes. The most likely mechanism of redistribution to produce the effect shown in Figure 15 is diffusion. Assuming a source for the hydrocarbons in the lower part of Lithologic Unit V, the concentration/ depth trends result from differences in effective diffusivities among the compounds considered. Ethane, as the most mobile compound, reveals the steepest gradient, that is, a major part of the section has already been enriched by ethane. An alternative, although unlikely, explanation for the observed effects could be the differences in water solubility of, for example, ethane and $n$ hexane [60 vs. $9.5 \mu \mathrm{g} / \mathrm{g}$, respectively, at ambient temper- ature, according to McAuliffe (1966)]. However, the light hydrocarbon concentrations in Hole 547A are so low that saturation in the pore water is never reached. The preferential transport of the more soluble species by upward-moving formation waters can, therefore, be ruled out.

Another possibility to explain the relative differences of the concentration depth trends in Figure 15 would be the assumption of an upward flux of a mobile liquid phase leading to a chromatographic separation due to a nonsteady state adsorption/desorption of the light hydrocarbons. It is difficult at this stage of our investigation to decide which single mechanism, or combination of different mechanisms, could best explain the observed phenomenon in this hole.

As an attempt to test the diffusion hypothesis, Figure 16 was prepared. The average concentration values for the nannofossil-bearing claystones analyzed between 722.0 and $771.7 \mathrm{~m}$ depth was set to $100 \%$ since this interval 
Table 1. (Continued).

\begin{tabular}{|c|c|c|c|c|c|c|c|c|}
\hline $\begin{array}{l}545-56-1,0-2 \\
521.5\end{array}$ & $\begin{array}{c}545-56-2,0-2 \\
523.0\end{array}$ & $\begin{array}{l}545-56-3,0-2 \\
524.5\end{array}$ & $\begin{array}{l}545-56-4,0-2 \\
526.0\end{array}$ & $\begin{array}{c}545-56-5,0-2 \\
527.5\end{array}$ & $\begin{array}{c}545-56-6,0-2 \\
529.0\end{array}$ & $\begin{array}{c}545-56-7,0-2 \\
530.5\end{array}$ & $\begin{array}{c}\text { 547A-5-4, } 142-144 \\
113.9\end{array}$ & $\begin{array}{c}547 \mathrm{~A}-10-2,0-2 \\
157.0\end{array}$ \\
\hline \multicolumn{2}{|c|}{$\begin{array}{c}\text { Nannofossil- } \\
\text { bearing } \\
\text { claystone }\end{array}$} & $\begin{array}{l}\text { Nannofossil- } \\
\text { bearing } \\
\text { calcareous } \\
\text { mudstone }\end{array}$ & $\begin{array}{c}\text { Nannofossil- } \\
\text { rich } \\
\text { calcareous } \\
\text { claystone }\end{array}$ & $\begin{array}{c}\text { Slightly } \\
\text { clayey } \\
\text { nannofossil } \\
\text { ooze, } \\
\text { pyrite }\end{array}$ & $\begin{array}{c}\text { Soft } \\
\text { muddy } \\
\text { dolomicro- } \\
\text { sporite }\end{array}$ & $\begin{array}{c}\text { Soft } \\
\text { microdolo- } \\
\text { sporite }\end{array}$ & $\begin{array}{l}\text { Slightly } \\
\text { clayey } \\
\text { nannofossil } \\
\text { ooze }\end{array}$ & $\begin{array}{l}\text { Nannofossil } \\
\text { ooze }\end{array}$ \\
\hline 0.95 & 1.01 & 0.47 & 0.79 & 0.01 & 0.10 & 0.01 & 0.01 & 0.16 \\
\hline 74 & 174 & 109 & 90 & $\mathrm{i}$ & $\mathrm{i}$ & $\mathrm{i}$ & $\mathrm{i}$ & 60 \\
\hline 115 & 108 & 264 & 166 & $\mathrm{i}$ & $\mathrm{i}$ & $\mathrm{i}$ & $\mathrm{i}$ & 575 \\
\hline 424 & 419 & 418 & 418 & 528 & $\mathrm{i}$ & 454 & $\mathrm{i}$ & 407 \\
\hline \multicolumn{9}{|c|}{ Concentration (ng/g dry sediment weight) } \\
\hline 1.9 & 2.7 & 2.4 & 1.4 & 0.92 & 0.53 & 2.6 & 1.8 & 1.2 \\
\hline 8.0 & 8.1 & 9.1 & 5.6 & 5.0 & 6.2 & 7.7 & 7.6 & 6.4 \\
\hline 1.8 & 2.2 & 3.4 & 1.4 & 1.1 & 1.1 & 3.2 & 1.6 & 1.1 \\
\hline 10.0 & 12.0 & 14.6 & 8.7 & 8.3 & 8.2 & 11.4 & 8.5 & 8.8 \\
\hline 3.2 & 5.9 & 6.0 & 3.6 & 2.9 & 2.7 & 3.6 & 2.1 & 2.5 \\
\hline 1.5 & 2.5 & 4.1 & 2.0 & 1.4 & 1.5 & 4.2 & 2.4 & 1.6 \\
\hline 6.4 & 9.3 & 9.6 & 4.9 & 4.2 & 4.3 & 5.8 & 4.6 & 4.9 \\
\hline 0.51 & 0.74 & 0.92 & 0.41 & 0.29 & 0.27 & 0.80 & 0.29 & 0.47 \\
\hline 0.14 & 0.34 & 0.17 & 0.09 & 0.03 & 0.06 & 0.05 & 0.06 & 0.12 \\
\hline 0.46 & 0.55 & 0.80 & 0.37 & 0.28 & 0.28 & 0.83 & 0.33 & 0.32 \\
\hline 7.5 & 13.4 & 5.8 & 4.4 & 1.4 & 1.5 & 2.1 & 1.3 & 1.8 \\
\hline 8.8 & 15.6 & 8.8 & 5.5 & 2.6 & 2.9 & 3.8 & 3.6 & 3.9 \\
\hline 0.18 & 0.52 & 0.23 & 0.14 & 0.02 & 0.05 & 0.02 & $<0.01$ & $<0.01$ \\
\hline 0.43 & 0.65 & 0.43 & 0.21 & $<0.1$ & 0.10 & 0.18 & 0.15 & $<0.1$ \\
\hline 0.43 & 1.1 & 0.41 & 0.29 & $<0.1$ & $<0.1$ & $<0.1$ & $<0.1$ & $<0.1$ \\
\hline 11.4 & 26.9 & 8.7 & 8.1 & 0.34 & 0.47 & 0.58 & 0.75 & 0.68 \\
\hline 1.4 & 3.0 & 2.0 & 1.1 & 0.27 & 0.36 & 1.0 & 0.57 & 0.63 \\
\hline 13.4 & 30.9 & 13.1 & 9.9 & 1.9 & 2.3 & 2.8 & 3.6 & 3.2 \\
\hline 1.3 & 2.3 & 1.2 & 0.65 & 0.12 & 0.27 & 0.3 & 0.62 & 0.28 \\
\hline 0.20 & 0.71 & 0.14 & 0.25 & $<0.1$ & $<0.1$ & $<0.1$ & $<0.1$ & $<0.1$ \\
\hline 2.8 & 4.0 & 11.8 & 2.9 & 2.0 & 1.3 & 2.6 & 2.3 & 1.2 \\
\hline 0.65 & 1.2 & 0.43 & 0.29 & $<0.1$ & $<0.1$ & $<0.1$ & 0.24 & $<0.1$ \\
\hline 2.0 & 5.7 & 2.2 & 2.0 & 0.11 & 0.18 & 0.17 & 0.36 & 0.30 \\
\hline 3.8 & 11.6 & 4.2 & 4.2 & $<0.1$ & $<0.1$ & $<0.1$ & 0.12 & $<0.1$ \\
\hline 1.7 & 4.6 & 1.9 & 1.7 & $<0.1$ & 0.18 & 0.17 & 0.28 & 0.27 \\
\hline 0.31 & 0.63 & 0.66 & 0.24 & $<0.1$ & 0.14 & 0.48 & 0.14 & 0.19 \\
\hline 0.31 & 0.68 & 0.26 & 0.22 & $<0.1$ & $<0.1$ & $<0.1$ & $<0.1$ & $<0.1$ \\
\hline 0.52 & 1.2 & 0.50 & 0.33 & $<0.1$ & $<0.1$ & $<0.1$ & $<0.1$ & $<0.1$ \\
\hline 20.8 & 67.6 & 25.5 & 25.0 & 3.3 & 2.1 & 2.7 & 2.4 & 2.1 \\
\hline 0.10 & $<0.1$ & 0.14 & $<0.1$ & $<0.1$ & - & $<0.1$ & $<0.1$ & $<0.1$ \\
\hline 1.7 & 4.4 & 1.6 & 1.2 & $<0.1$ & 0.11 & $<0.1$ & 0.12 & 0.22 \\
\hline$<0.1$ & 0.42 & 0.14 & 0.24 & - & $<0.1$ & $<0.1$ & $<0.1$ & $<0.1$ \\
\hline 0.50 & 1.8 & 0.55 & 0.72 & $<0.1$ & $<0.1$ & $<0.1$ & $<0.1$ & $<0.1$ \\
\hline 0.10 & 0.59 & $<0.1$ & $<0.1$ & $<0.1$ & $<0.1$ & - & $<0.1$ & - \\
\hline 0.41 & 1.4 & 0.41 & 0.29 & $<0.1$ & $<0.1$ & - & $<0.1$ & - \\
\hline 11.5 & 24.2 & 69.0 & 6.0 & 3.9 & 2.1 & 6.0 & 5.6 & 5.6 \\
\hline 4.8 & 39.4 & 15.6 & 21.6 & $<0.1$ & $<0.1$ & $<0.1$ & 0.12 & 0.22 \\
\hline 6.4 & 67.6 & 34.2 & 49.2 & 0.76 & 0.81 & 1.7 & 2.4 & 3.6 \\
\hline
\end{tabular}

appears to bear the unmodified, in situ generated light hydrocarbon concentrations appropriate for this maturity level. The concentrations of all shallower samples were expressed as the percentage of this unmodified level, and smoothed depth trends were drawn in Figure 16 for individual compounds. The uppermost sample still contains about $40 \%$ of the unmodified ethane concentration, and for propane the value is about $10 \%$. For $n$ pentane, $n$-hexane, and $n$-heptane only a few percent are reached in the shallowest samples. The strong differences between, for example, ethane and $n$-pentane, shown on the left of Figure 16, are most probably caused by differences in the diffusivities of these compounds. Effective diffusion coefficients for migration of light hydrocarbons through the water-saturated pore space were found to decrease exponentially with increasing carbon number (Leythaeuser et al., 1982). Previously, the effects of light hydrocarbon diffusion through sedimentary rocks have been documented to occur only over short (about $10 \mathrm{~m}$ ) or intermediate (about $180 \mathrm{~m}$ ) depth ranges (Leythaeuser et al., 1980, 1983; Thompson, 1979, respectively). The 700-m-thick interval studied here represents a documentation for the effects of possible diffusion processes in the sedimentary column over a considerable vertical distance. In Hole 547A benzene shows a depth trend similar to that of $n$-butane (not shown in Fig. 16). This is surprising since the effective diffusion coefficient of benzene, because of its high polarity, is expected to be much lower than that of $n$-butane. The behavior of benzene cannot be explained at present. It possibly reflects a different transport mechanism from that of the $n$-alkanes.

Other parameters to document the relative enrichment of the upper interval of Site 547 by gaseous hydrocarbons are illustrated in Figure 17. Elevated values for the $\mathrm{C}_{2}-\mathrm{C}_{5}$ hydrocarbon content (in \%) and the ethane/nheptane ratio are observed for samples from Lithologic Unit I to Subunit IVB, as well as for one sample of Unit 
Table 1. (Continued).

\begin{tabular}{|c|c|c|c|c|c|c|}
\hline & $\begin{array}{l}\text { Sample (interval in } \mathrm{cm} \text { ) } \\
\text { Depth (m) }\end{array}$ & $\begin{array}{c}\text { Clayey } \\
\text { nannofossil } \\
\text { ooze }\end{array}$ & $\begin{array}{c}\text { 547A-20-2, 0-2 } \\
252.0 \\
\text { Clayey } \\
\text { nannofossil } \\
\text { ooze, } \\
\text { nannofossil } \\
\text { chalk } \\
\text { clasts }\end{array}$ & $\begin{array}{c}\text { 547A-25-1, 52-53 } \\
298.5 \\
\text { Siliceous } \\
\text { clayey } \\
\text { nannofossil } \\
\text { chalk, } \\
\text { burrows }\end{array}$ & $\begin{array}{c}\text { 547A-30-3, 0-2 } \\
348.5 \\
\text { Slightly } \\
\text { clayey } \\
\text { nannofossil } \\
\text { chalk, } \\
\text { bioturbated }\end{array}$ & $\begin{array}{l}\text { Clayey } \\
\text { nannofossil } \\
\text { chalk }\end{array}$ \\
\hline & $\begin{array}{l}\mathrm{C}_{\text {org }}[\% \text { dry sediment weight }] \\
\text { Hydrogen index }[\mathrm{mg} \text { hydrocarbons } / \mathrm{g} \mathrm{C} \text { org] } \\
\text { Oxygen index }\left[\mathrm{mg} \mathrm{CO}_{2} / \mathrm{g} \mathrm{C}_{\text {org }}\right] \\
T_{\max }\left[{ }^{\circ} \mathrm{C}\right]\end{array}$ & $\begin{array}{c}0.01 \\
i \\
i \\
424\end{array}$ & $\begin{array}{l}0.99 \\
220 \\
167 \\
421\end{array}$ & $\begin{array}{l}0.24 \\
16 \\
320 \\
422\end{array}$ & $\begin{array}{c}0.01 \\
\mathrm{i} \\
\mathrm{i} \\
432\end{array}$ & $\begin{array}{l}0.11 \\
\text { n.d. } \\
\text { n.d. } \\
\text { n.d. }\end{array}$ \\
\hline Ref. No. & Hydrocarbon & & Concentrat & ion $(\mathrm{ng} / \mathrm{g}$ dry sedim & nt weight) & \\
\hline 1 & Ethene & 2.2 & 2.5 & 3.3 & 2.5 & 2.5 \\
\hline 2 & Ethane & 5.8 & 12.1 & 11.6 & 12.7 & 15.4 \\
\hline 3 & Propene & 2.0 & 3.8 & 5.7 & 3.6 & 2.8 \\
\hline 4 & Propane & 8.6 & 16.6 & 14.2 & 17.3 & 24.3 \\
\hline 5 & Methylpropane & 3.0 & 7.2 & 4.8 & 4.6 & 7.7 \\
\hline 6 & Methylpropene + 1-butene & 2.9 & 6.2 & 5.5 & 4.5 & 5.4 \\
\hline 7 & $n$-Butane & 4.4 & 8.9 & 7.5 & 8.7 & 11.7 \\
\hline 8 & trans-2-Butene & 0.74 & 1.0 & 1.5 & 0.92 & 1.4 \\
\hline 9 & 2,2-Dimethylpropane & 0.05 & 0.25 & 0.10 & 0.13 & 0.13 \\
\hline 10 & cis-2-Butene & 0.74 & 0.84 & 1.4 & 0.83 & 1.3 \\
\hline 11 & Methylbutane & 1.5 & 4.0 & 2.6 & 2.9 & 4.3 \\
\hline 12 & $n$-Pentane & 3.6 & 7.3 & 5.7 & 5.7 & 7.8 \\
\hline 13 & 2,2-Dimethylbutane & 0.02 & $<0.01$ & 0.08 & $<0.01$ & $<0.01$ \\
\hline 14 & Cyclopentane & 0.13 & 0.28 & 0.30 & 0.21 & 0.37 \\
\hline 15 & 2,3-Dimethylbutane & $<0.1$ & 0.37 & 0.16 & - & $<0.1$ \\
\hline 16 & 2-Methylpentane & 0.63 & 2.8 & 1.4 & 1.8 & 1.5 \\
\hline 17 & 3-Methylpentane & 0.84 & 2.4 & 1.2 & 0.86 & 1.2 \\
\hline 18 & $n$-Hexane & 2.7 & 9.4 & 4.3 & 4.1 & 5.2 \\
\hline 19 & Methylcyclopentane + 2,2-dimethylpentane & 0.22 & 1.6 & 1.4 & 0.58 & 0.68 \\
\hline 20 & 2,4-Dimethylpentane & $<0.1$ & 0.17 & 0.13 & 0.10 & $<0.1$ \\
\hline 21 & Benzene & 2.6 & 2.3 & 4.3 & 3.0 & 3.7 \\
\hline 22 & Cyclohexane & 0.11 & 0.81 & 0.33 & $<0.1$ & 0.12 \\
\hline 23 & 2-Methylhexane & 0.22 & 1.1 & 0.57 & 0.42 & 0.45 \\
\hline 24 & 2,3-Dimethylpentane + 1,1-dimethylcyclopentane & 0.11 & 0.58 & 0.27 & 0.12 & 0.31 \\
\hline 25 & 3-Methylhexane & 0.22 & 1.0 & 0.58 & 0.32 & 0.41 \\
\hline 26 & 1,cis-3-Dimethylcyclopentane & 0.28 & 0.34 & 0.34 & 0.29 & 0.57 \\
\hline 27 & 1,trans-3-Dimethylcyclopentane & $<0.1$ & 0.17 & 0.16 & $<0.1$ & $<0.1$ \\
\hline 28 & 1,trans-2-Dimethylcyclopentane & $<0.1$ & 0.14 & 0.18 & 0.13 & 0.29 \\
\hline 29 & $n$-Heptane & 2.1 & 5.2 & 2.8 & 2.5 & 3.8 \\
\hline 30 & 1, cis-2-Dimethylcyclopentane & $<0.1$ & $<0.1$ & $<0.1$ & $<0.1$ & $<0.1$ \\
\hline 31 & Methylcyclohexane & 0.14 & 0.51 & 0.52 & 0.26 & 0.29 \\
\hline 32 & 2,5-Dimethylhexane & - & - & - & $<0.1$ & $<0.1$ \\
\hline 33 & Ethylcyclopentane & $<0.1$ & 0.17 & $<0.1$ & $<0.1$ & 0.12 \\
\hline 34 & 1,trans-2,cis-4-Trimethylcyclopentane & - & - & - & $<0.1$ & - \\
\hline 35 & 1,trans-2,cis-3-Trimethylcyclopentane & - & - & - & $<0.1$ & - \\
\hline 36 & Toluene & 2.6 & 18.6 & 8.0 & 8.2 & 12.0 \\
\hline 37 & 2-Methylheptane & $<0.1$ & 0.75 & 0.13 & 0.13 & 0.44 \\
\hline 38 & $n$-Octane & 1.5 & 4.1 & 1.3 & 1.9 & 2.5 \\
\hline
\end{tabular}

V (Sample 547B-4-2, 0-2 cm) and for several samples from Subunit VIA. The depth patterns shown in this figure are similarly, as discussed above, explained as the result of diffusion processes. Gaseous hydrocarbons were also redistributed from the lower part of Lithologic Unit $\mathrm{V}$ and transported into the underlying Jurassic limestone conglomerate (Subunit VIA) that has a higher porosity. Ethane/ $n$-heptane ratios in excess of 5.0 are observed here.

The toluene/ $\mathrm{C}_{7}$-cycloalkane concentration ratio in Figure 18 remains fairly constant with depth and below 1.0 in Lithologic Unit V and increases sharply at the contacts with the other lithologic units above and below. This relative enrichment of toluene is tentatively explained, as for Site 545 , by the assumption of compaction water flow through sediments of Unit I to Subunits IVB and VIA and the preferential adsorption of this compound on the mineral surfaces.
Variations in the isoalkane/ $n$-alkane ratios (for $\mathrm{C}_{4}$ and $\mathrm{C}_{5}$ ) are also found at Site 547 (Fig. 19). Changes in lithology and type of organic matter cause variations in the methylpropane/ $n$-butane ratio by a factor of two and in the methylbutane/ $n$-pentane ratio by a factor of four over the entire depth interval. Therefore, these concentration ratios cannot be considered universally suitable for an assessment of the maturity stage in low-mature sediments (cf. Hunt et al., 1980b). Instead, they appear to be strongly lithology dependent and, possibly, influenced by migration processes. The strong variation within Lithologic Unit V, however, cannot be conclusively explained at present (see discussion of the corresponding data at Site 545).

In a similar sense we interpret the depth trends for the relative compositions of the $\mathrm{C}_{5}$ and $\mathrm{C}_{7}$ hydrocarbons (Fig. 20). The higher relative proportion of cyclopentane (marked N5 on the left of Fig. 20) in Unit V (mean 
Table 1. (Continued).

\begin{tabular}{|c|c|c|c|c|c|c|c|}
\hline $\begin{array}{c}547 \mathrm{~A}-41-1,148-150 \\
442.0\end{array}$ & $\begin{array}{c}547 \mathrm{~A}-45-2,0-2 \\
480.0\end{array}$ & $\begin{array}{c}547 \mathrm{~A}-50-2,0-2 \\
526.0\end{array}$ & $\begin{array}{c}\text { 547A-55-2, } 0-2 \\
575.0\end{array}$ & $\begin{array}{c}547 \mathrm{~A}-60-4,138-140 \\
626.9\end{array}$ & $\begin{array}{c}547 \mathrm{~A}-66-2,0-2 \\
679.5\end{array}$ & $\begin{array}{c}\text { 547A-71-2, } 0-1 \\
722.0\end{array}$ & $\begin{array}{l}\text { 547B-4-2, } 0-2 \\
\quad 754.5\end{array}$ \\
\hline Claystone & $\begin{array}{c}\text { Nannofossil- } \\
\text { bearing } \\
\text { claystone, } \\
\text { burrows }\end{array}$ & \multicolumn{3}{|c|}{$\begin{array}{c}\text { Nannofossil- } \\
\text { bearing } \\
\text { claystone }\end{array}$} & $\begin{array}{c}\text { Nannofossil- } \\
\text { bearing } \\
\text { mudstone, } \\
\text { burrows }\end{array}$ & $\begin{array}{l}\text { Nannofossil- } \\
\text { bearing } \\
\text { claystone }\end{array}$ & $\begin{array}{l}\text { Slightly } \\
\text { calcareous } \\
\text { mudstone/ } \\
\text { claystone }\end{array}$ \\
\hline 0.69 & 0.38 & 0.67 & 0.61 & 0.50 & 1.08 & 0.73 & 0.72 \\
\hline 38 & 47 & 49 & 41 & 59 & 141 & 122 & 96 \\
\hline 161 & 244 & 214 & 220 & 389 & 212 & 236 & 567 \\
\hline 419 & 417 & 417 & 412 & 420 & 427 & 426 & 427 \\
\hline \multicolumn{8}{|c|}{ Concentration (ng/g dry sediment weight) } \\
\hline 2.5 & 2.5 & 2.8 & 3.1 & 3.2 & 1.9 & 2.6 & 4.5 \\
\hline 10.5 & 11.2 & 19.4 & 10.7 & 20.4 & 28.7 & 19.3 & 25.4 \\
\hline 3.6 & 3.1 & 3.7 & 4.3 & 5.9 & 3.1 & 3.5 & 6.5 \\
\hline 18.4 & 31.3 & 59.1 & 51.7 & 67.5 & 123 & 104 & 48.1 \\
\hline 9.4 & 16.5 & 38.3 & 38.8 & 52.5 & 90.4 & 85.0 & 20.0 \\
\hline 4.1 & 3.2 & 4.8 & 4.0 & 5.8 & 3.3 & 4.1 & 11.7 \\
\hline 14.4 & 24.8 & 49.9 & 41.5 & 53.0 & 91.9 & 91.3 & 24.8 \\
\hline 1.3 & 1.9 & 2.7 & 2.0 & 2.5 & 2.4 & 2.2 & 2.5 \\
\hline 0.23 & 0.24 & 0.40 & 0.35 & 0.42 & 0.69 & 0.63 & 0.18 \\
\hline 0.94 & 1.1 & 1.6 & 1.4 & 1.9 & 1.4 & 0.30 & 2.0 \\
\hline 27.6 & 47.3 & 110 & 93.5 & 107 & 161 & 180 & 22.0 \\
\hline 29.7 & 40.8 & 78.7 & 55.9 & 65.0 & 99.7 & 123 & 19.5 \\
\hline$<0.01$ & $<0.01$ & 1.0 & 0.27 & 0.46 & $<0.01$ & 0.89 & 0.09 \\
\hline 2.5 & 4.5 & 11.3 & 11.1 & 14.5 & 25.1 & 26.2 & 3.1 \\
\hline 2.5 & 3.4 & 6.9 & 4.0 & 4.0 & 4.7 & 6.2 & 0.47 \\
\hline 27.7 & 30.3 & 60.5 & 40.5 & 43.9 & 60.5 & 88.6 & 7.1 \\
\hline 4.7 & 5.1 & 10.0 & 6.5 & 7.1 & 10.8 & 16.3 & 3.7 \\
\hline 31.2 & 27.0 & 51.6 & 33.1 & 40.1 & 58.2 & 90.5 & 9.6 \\
\hline 18.0 & 25.5 & 60.1 & 45.3 & 50.0 & 79.1 & 100 & 7.9 \\
\hline 1.8 & 1.6 & 3.1 & 2.1 & 2.1 & 2.4 & 3.9 & $<0.1$ \\
\hline 5.8 & 8.4 & 17.1 & 17.9 & 19.8 & 35.7 & 35.8 & 12.6 \\
\hline 2.0 & 2.4 & 5.2 & 3.9 & 4.6 & 7.3 & 9.3 & 0.86 \\
\hline 5.1 & 3.9 & 7.2 & 4.6 & 5.4 & 7.0 & 13.2 & 0.90 \\
\hline 9.7 & 8.7 & 15.8 & 9.7 & 9.9 & 12.5 & 19.5 & 1.1 \\
\hline 4.9 & 3.9 & 6.9 & 4.3 & 4.8 & 6.5 & 11.9 & 0.53 \\
\hline 14.8 & 16.6 & 33.1 & 18.8 & 15.2 & 17.4 & 26.0 & 1.6 \\
\hline 15.7 & 18.3 & 41.0 & 25.0 & 20.6 & 22.5 & 32.6 & 0.90 \\
\hline 21.7 & 23.3 & 44.8 & 22.6 & 17.0 & 19.7 & 28.2 & 1.1 \\
\hline 31.7 & 21.2 & 42.0 & 28.6 & 38.4 & 45.9 & 82.4 & 7.7 \\
\hline 5.4 & 6.3 & 15.0 & 9.8 & 7.7 & 9.5 & 13.9 & 0.56 \\
\hline 18.8 & 20.2 & 44.6 & 27.3 & 24.7 & 31.3 & 39.6 & 3.9 \\
\hline 0.49 & 0.22 & 0.69 & 0.56 & 0.57 & 0.76 & 1.2 & $<0.1$ \\
\hline 5.1 & 4.8 & 10.1 & 6.1 & 6.1 & 8.8 & 14.5 & 0.56 \\
\hline 5.7 & 4.4 & 7.3 & 3.5 & 2.6 & 3.0 & 4.9 & $<0.1$ \\
\hline 2.3 & 2.1 & 2.8 & 1.7 & 0.99 & 3.0 & 3.6 & $<0.1$ \\
\hline 17.0 & 15.9 & 36.7 & 25.7 & 11.8 & 95.1 & 29.2 & 2.3 \\
\hline 9.4 & 5.4 & 11.3 & 7.7 & 10.4 & 13.6 & 20.9 & 0.56 \\
\hline 17.3 & 9.6 & 21.3 & 17.8 & 23.8 & 22.8 & 29.2 & 1.1 \\
\hline
\end{tabular}

value $6.7 \%$ ), however, as compared to Unit III from Site 545 (Fig. 9), can be explained as a maturity effect. This relative increase of the cyclopentane concentration with increasing maturity is just the opposite from what has been frequently observed and is predicted from thermodynamic considerations for advanced maturity stages (that is, after the intense hydrocarbon generation is initiated; Philippi, 1975; Schaefer et al., in press).

The changes in the relative composition of the $\mathrm{C}_{7}$-hydrocarbons (shown on the right of Fig. 20) reflect the combined effect of changes in lithology and source material as well as redistribution processes, whereby the latter had the more drastic influence on the composition, particularly being obvious from the depth variations of the toluene content.

\section{CONCLUSIONS}

The occurrence of essentially nonbiogenic low-molecular-weight hydrocarbons is a very sensitive indicator for the thermally controlled hydrocarbon-generation reactions of kerogen. Furthermore, these compounds are more water soluble than their higher homologs, and there are greater differences in their individual mobilities (e.g., by diffusion) within the water-saturated pore spaces of the sediment. Therefore, light hydrocarbon distributions represent a valuable tool to study generation and migration processes.

1. Differences in the averaged total light hydrocarbon concentrations $\left(\mathrm{C}_{2}-\mathrm{C}_{8}\right)$ between Site 545 (Lithologic Subunits IIIA and IIIB: $3.2 \times 10^{4} \mathrm{ng} / \mathrm{g} \mathrm{C}_{\text {org }}$ ) and Site 547 (Unit V: $1.0 \times 10^{5} \mathrm{ng} / \mathrm{g} \mathrm{C}_{\text {org }}$ ) indicate a slightly more advanced maturity stage at Site 547 possibly due to its proximity to a salt diapir. Both values are typical, however, for immature organic matter in terms of the classical hydrocarbon-generation concept.

2. Values in excess of $10^{5} \mathrm{ng} / \mathrm{g} \mathrm{C}_{\text {org }}$ at Site 545 and of $2 \times 10^{5} \mathrm{ng} / \mathrm{g} \mathrm{C}_{\text {org }}$ at Site 547 for the organic-carbonlean samples $\left(\mathrm{C}_{\text {org }}<0.1 \%\right)$ indicate a slight enrichment 
Table 1. (Continued).

\begin{tabular}{|c|c|c|c|c|c|c|}
\hline & $\begin{array}{l}\text { Sample (interval in cm) } \\
\text { Depth (m) }\end{array}$ & $\begin{array}{c}\text { 547B-5-1, 11-13 } \\
762.6\end{array}$ & $\begin{array}{c}547 \mathrm{~B}-5-2,70-72 \\
764.7\end{array}$ & $\begin{array}{c}547 \mathrm{~B}-5-3,104-106 \\
766.6\end{array}$ & $\begin{array}{c}547 \mathrm{~B}-5-4,58-60 \\
767.6\end{array}$ & $\begin{array}{c}\text { 547B-5-5, 58-60 } \\
769.1\end{array}$ \\
\hline & Lithology & \multicolumn{5}{|c|}{$\begin{array}{c}\text { Nannofossil- } \\
\text { bearing } \\
\text { claystone }\end{array}$} \\
\hline & $\mathrm{C}_{\text {org }}[\%$ dry sediment weight] & 2.31 & 0.90 & 1.13 & 1.11 & 1.07 \\
\hline & Hydrogen index [ $\mathrm{mg}$ hydrocarbons/g $\mathrm{C}_{\mathrm{org}}$ ] & 127 & 133 & 105 & 120 & 135 \\
\hline & Oxygen index [mg $\mathrm{CO}_{2} / \mathrm{g} \mathrm{C}_{\text {org }}$ ] & 97 & 184 & 206 & 99 & 133 \\
\hline & $T_{\max }\left[{ }^{\circ} \mathrm{C}\right]$ & 427 & 428 & 429 & 428 & 428 \\
\hline Ref. No. & Hydrocarbon & \multicolumn{5}{|c|}{ Concentration (ng/g dry sediment weight) } \\
\hline 1 & Ethene & 2.0 & 3.5 & 3.8 & 2.5 & 2.4 \\
\hline 2 & Ethane & 9.0 & 17.7 & 15.6 & 13.4 & 16.5 \\
\hline 3 & Propene & 1.7 & 3.2 & 2.1 & 2.5 & 2.3 \\
\hline 4 & Propane & 61.7 & 81.7 & 26.7 & 60.1 & 73.1 \\
\hline 5 & Methylpropane & 57.2 & 76.5 & 16.1 & 48.4 & 57.7 \\
\hline 6 & Methylpropene + 1-butene & 3.0 & 4.2 & 2.2 & 3.4 & 3.9 \\
\hline 7 & $n$-Butane & 70.8 & 93.3 & 18.6 & 58.3 & 68.5 \\
\hline 8 & trans-2-Butene & 1.5 & 2.0 & 0.56 & 1.5 & 1.5 \\
\hline 9 & 2,2-Dimethylpropane & 0.70 & 0.94 & 0.24 & 0.54 & 0.67 \\
\hline 10 & cis-2-Butene & 0.81 & 1.3 & 0.48 & 0.96 & 0.86 \\
\hline 11 & Methylbutane & 138 & 183 & 67.5 & 101 & 127 \\
\hline 12 & $n$-Pentane & 104 & 143 & 57.7 & 77.7 & 94.3 \\
\hline 13 & 2,2-Dimethylbutane & 0.77 & 1.1 & 0.58 & $<0.01$ & $<0.01$ \\
\hline 14 & Cyclopentane & 18.8 & 23.2 & 11.0 & 13.6 & 16.2 \\
\hline 15 & 2,3-Dimethylbutane & 6.2 & 8.6 & 5.3 & 4.6 & 6.1 \\
\hline 16 & 2-Methylpentane & 78.1 & 112 & 72.1 & 56.8 & 74.2 \\
\hline 17 & 3-Methylpentane & 13.9 & 20.2 & 14.1 & 11.0 & 14.8 \\
\hline 18 & $n$-Hexane & 81.2 & 117 & 88.8 & 58.4 & 74.3 \\
\hline 19 & Methylcyclopentane + 2,2-dimethylpentane & 79.0 & 106 & 80.9 & 56.6 & 74.5 \\
\hline 20 & 2,4-Dimethylpentane & 3.1 & 4.7 & 4.1 & 2.4 & 3.4 \\
\hline 21 & Benzene & $<0.1$ & 48.4 & 17.3 & 13.2 & 23.2 \\
\hline 22 & Cyclohexane & 7.6 & 10.6 & 8.5 & 5.4 & 6.9 \\
\hline 23 & 2-Methylhexane & 11.5 & 16.6 & 15.4 & 8.1 & 11.4 \\
\hline 24 & 2,3-Dimethylpentane + 1,1-dimethylcyclopentane & 19.3 & 28.2 & 26.7 & 15.1 & 22.7 \\
\hline 25 & 3-Methylhexane & 10.5 & 15.3 & 15.0 & 8.0 & 12.3 \\
\hline 26 & 1,cis-3-Dimethylcyclopentane & 15.0 & 20.6 & 18.6 & 10.9 & 14.7 \\
\hline 27 & 1,trans-3-Dimethylcyclopentane & 16.8 & 23.0 & 21.1 & 11.9 & 16.7 \\
\hline 28 & 1,trans-2-Dimethylcyclopentane & 20.0 & 28.8 & 27.8 & 16.1 & 24.7 \\
\hline 29 & $n$-Heptane & 96.1 & 138 & 119 & 62.4 & 82.4 \\
\hline 30 & 1,cis-2-Dimethylcyclopentane & 8.9 & 11.5 & 11.3 & 6.4 & 9.0 \\
\hline 31 & Methylcyclohexane & 55.5 & 78.0 & 81.5 & 45.6 & 69.5 \\
\hline 32 & 2,5-Dimethylhexane & 1.9 & 1.1 & 2.1 & 1.0 & 1.5 \\
\hline 33 & Ethylcyclopentane & 13.9 & 14.4 & 16.8 & 8.9 & 13.4 \\
\hline 34 & 1,trans-2,cis-4-Trimethylcyclopentane & 8.5 & 4.2 & 6.1 & 2.9 & 5.8 \\
\hline 35 & 1,trans-2,cis-3-Trimethylcyclopentane & 8.5 & 3.5 & 6.9 & 4.1 & 8.4 \\
\hline 36 & Toluene & 47.6 & 96.0 & 62.0 & 43.7 & 47.4 \\
\hline 37 & 2-Methylheptane & 29.1 & 32.8 & 41.8 & 19.1 & 27.9 \\
\hline 38 & $n$-Octane & 54.6 & 51.8 & 70.8 & 35.5 & 40.0 \\
\hline
\end{tabular}

by gaseous hydrocarbons migrating through the sedimentary column.

3. Comparing the concentration versus depth trends for individual hydrocarbons leads to the conclusion that significant movement of the gaseous hydrocarbons, particularly ethane, occurred from the claystones at Site 545 (Lithologic Subunits IIIA and IIIB) to the adjacent nannofossil chalks and oozes (Subunit IB and Unit II as well as Subunit IIIC). Moreover, it appears that the chalk section (Unit II), possibly by movement of formation waters, was depleted initially in total light hydrocarbons and subsequently replenished by migrating gases. This conclusion is based on a comparison of light hydrocarbon concentrations between two samples of different lithology just above and below the Neogene/mid-Cretaceous unconformity.

4. Individual $n$-alkane concentrations for Hole 547A plotted versus depth exhibit steeper gradients with decreasing carbon number of the $n$-alkanes. This is just the opposite of what would be expected if this effect would be caused purely by hydrocarbon-generation processes. The most likely explanation for the phenomenon is the redistribution by diffusion of the most mobile compounds from the relatively organic-rich section in Unit $\mathrm{V}$, that is, from below $700 \mathrm{~m}$ up toward the surface.

5. The relative concentration of cyclopentane (as a proportion of total $\mathrm{C}_{5}$ hydrocarbons) has a mean value of 3.0\% in the nannofossil claystones of Unit III at Site 545 , whereas in Unit V at Site 547 (similar lithology) a mean value of $6.7 \%$ is measured. Obviously this parameter is increasing with thermal evolution in the very early stages of hydrocarbon generation. It is known, however, that this parameter decreases again during intense hydrocarbon generation with maturation progress.

6. Strong relative enrichment by toluene in Lithologic Subunits IA, IB, and Unit II as well as in the lower part of Unit III (Site 545) and in Unit I to Subunits IVB and VIA (Site 547 ) is tentatively explained by a higher 
Table 1. (Continued).

\begin{tabular}{|c|c|c|c|c|c|c|c|}
\hline $\begin{array}{c}547 \mathrm{~B}-5-6,53-55 \\
770.5\end{array}$ & $\begin{array}{c}\text { 547B-5-7, 20-22 } \\
771.7\end{array}$ & $\begin{array}{c}\text { 547B-6-1, } 44-46 \\
772.5\end{array}$ & $\begin{array}{c}\text { 547B-6-1, 113-115 } \\
773.1\end{array}$ & $\begin{array}{c}547 \mathrm{~B}-6-2,141-143 \\
774.9\end{array}$ & $\begin{array}{c}547 \mathrm{~B}-6-3,29-31 \\
775.3\end{array}$ & $\begin{array}{c}547 \mathrm{~B}-6-3,107-109 \\
776.1\end{array}$ & $\begin{array}{c}547 \mathrm{~B}-6-4,25-27 \\
776.8\end{array}$ \\
\hline & $\begin{array}{c}\text { Nannofossil- } \\
\text { bearing } \\
\text { claystone }\end{array}$ & & \multicolumn{5}{|c|}{$\begin{array}{c}\text { Limestone } \\
\text { conglomerate }\end{array}$} \\
\hline 1.17 & 1.74 & 0.77 & 0.36 & 0.01 & 0.01 & 0.01 & 0.01 \\
\hline 111 & 623 & 181 & i & i & i & $\mathrm{i}$ & $\mathrm{i}$ \\
\hline 90 & 324 & 334 & i & $\mathrm{i}$ & i & $\mathrm{i}$ & $\mathrm{i}$ \\
\hline 429 & 426 & 419 & 449 & i & i & $\mathrm{i}$ & $\mathrm{i}$ \\
\hline \multicolumn{8}{|c|}{ Concentration (ng/g dry sediment weight) } \\
\hline 2.9 & 1.6 & 4.0 & 2.5 & 2.9 & 2.1 & 1.0 & 1.3 \\
\hline 22.2 & 10.0 & 7.8 & 4.2 & 8.4 & 3.5 & 5.9 & 5.5 \\
\hline 3.0 & 1.6 & 3.5 & 2.7 & 4.2 & 2.4 & 1.1 & 1.5 \\
\hline 93.2 & 97.3 & 24.6 & 6.5 & 12.0 & 4.0 & 5.9 & 6.7 \\
\hline 67.9 & 72.0 & 30.1 & 1.5 & 3.6 & 1.3 & 1.6 & 1.7 \\
\hline 4.5 & 2.8 & 5.8 & 5.7 & 4.6 & 2.6 & 1.7 & 2.2 \\
\hline 81.3 & 83.1 & 33.9 & 3.2 & 5.0 & 1.7 & 2.8 & 3.2 \\
\hline 1.9 & 1.3 & 1.3 & 0.65 & 1.2 & 0.47 & 0.32 & 0.37 \\
\hline 0.73 & 0.76 & 0.42 & 0.05 & $<0.01$ & $<0.01$ & $<0.01$ & 0.03 \\
\hline 1.2 & 0.68 & 1.1 & 0.59 & 0.97 & 0.45 & 0.27 & 0.32 \\
\hline 139 & 146 & 72.8 & 1.4 & 1.9 & 0.58 & 0.85 & 0.93 \\
\hline 107 & 113 & 57.5 & 2.8 & 4.0 & 1.4 & 2.1 & 2.2 \\
\hline 0.90 & $<0.01$ & 0.54 & 0.05 & 0.15 & 0.23 & $<0.01$ & $<0.01$ \\
\hline 18.0 & 20.6 & 9.0 & 0.19 & 0.12 & $<0.1$ & $<0.1$ & $<0.1$ \\
\hline 7.0 & 7.9 & 4.9 & $<0.1$ & $<0.1$ & $<0.1$ & $<0.1$ & $<0.1$ \\
\hline 82.9 & 92.7 & 51.3 & 0.55 & 0.65 & 0.28 & 0.30 & 0.30 \\
\hline 17.2 & 19.3 & 11.5 & 0.54 & 0.98 & 0.50 & 0.34 & 0.30 \\
\hline 82.5 & 93.0 & 51.2 & 2.0 & 3.1 & 1.3 & 2.0 & 1.8 \\
\hline 81.7 & 97.7 & 49.6 & 0.36 & 0.27 & 0.17 & 0.20 & 0.18 \\
\hline 3.9 & 4.6 & 3.1 & $<0.1$ & $<0.1$ & $<0.1$ & $<0.1$ & $<0.1$ \\
\hline 20.4 & 34.1 & 9.6 & $<0.1$ & $<0.1$ & $<0.1$ & $<0.1$ & $<0.1$ \\
\hline 7.8 & 8.9 & 4.5 & $<0.1$ & $<0.1$ & $<0.1$ & $<0.1$ & $<0.1$ \\
\hline 12.6 & 14.9 & 8.9 & 0.22 & 0.28 & 0.12 & 0.17 & 0.15 \\
\hline 25.8 & 31.5 & 20.9 & 0.18 & 0.20 & $<0.1$ & $<0.1$ & $<0.1$ \\
\hline 14.4 & 17.7 & 12.0 & $<0.1$ & 0.20 & 0.12 & 0.17 & $<0.1$ \\
\hline 16.3 & 19.6 & 10.9 & 0.27 & 0.60 & 0.17 & 0.17 & 0.15 \\
\hline 18.5 & 23.1 & 13.5 & $<0.1$ & $<0.1$ & $<0.1$ & $<0.1$ & $<0.1$ \\
\hline 28.3 & 35.8 & 22.3 & $<0.1$ & $<0.1$ & $<0.1$ & $<0.1$ & $<0.1$ \\
\hline 130 & 105 & 55.3 & 3.4 & 3.0 & 1.7 & 0.99 & 1.05 \\
\hline 9.5 & 11.8 & 5.7 & $<0.1$ & $<0.1$ & $<0.1$ & $<0.1$ & $<0.1$ \\
\hline 78.0 & 96.4 & 55.9 & 0.22 & $<0.1$ & $<0.1$ & $<0.1$ & $<0.1$ \\
\hline 1.6 & 2.0 & 1.2 & $<0.1$ & $<0.1$ & $<0.1$ & $<0.1$ & $<0.1$ \\
\hline 14.3 & 19.0 & 11.1 & $<0.1$ & $<0.1$ & $<0.1$ & $<0.1$ & $<0.1$ \\
\hline 7.6 & 12.1 & 8.7 & - & $<0.1$ & - & $<0.1$ & $<0.1$ \\
\hline 10.9 & 14.9 & 13.0 & - & $<0.1$ & - & $<0.1$ & $<0.1$ \\
\hline 40.6 & 70.3 & 50.9 & 1.7 & 4.9 & 2.6 & 0.60 & 0.59 \\
\hline 34.5 & 37.2 & 21.6 & $<0.1$ & $<0.1$ & $<0.1$ & $<0.1$ & - \\
\hline 52.5 & 67.8 & 27.5 & 0.78 & 2.8 & 1.9 & 0.54 & 0.44 \\
\hline
\end{tabular}

rate of movement of hydrocarbon-bearing formation waters and the preferential adsorption of polar compounds on the mineral surfaces.

\section{ACKNOWLEDGMENTS}

The supply of samples with the assistance of the U.S. National Science Foundation and financial support by the Deutsche Forschungsgemeinschaft, Schwerpunkt "Deep Sea Drilling Project" (DFG Grant No. Le 469/1) are gratefully acknowledged.

We also thank Dr. J. Rullkötter, KFA-Jülich, who participated as Organic Geochemist on DSDP Leg 79 and took the excellent sample set and who provided the detailed lithologic description of the samples. For extensive technical assistance we are indebted to Mrs. M. Derichs and B. Winden as well as Messrs. U. Disko, J. Höltkemeier, H. Pooch, and H. G. Sittardt, and to Mrs. A. Köntges who typed the manuscript (all at KFA-Jülich).

The authors are most grateful for detailed reviews by Dr. C. Cornford (Glasgow, Scotland) and Dr. K. Kvenvolden (Menlo Park, California, U.S.A.).

\section{REFERENCES}

Boutefeu, A., Leplat, P., and Somers, Y., 1980. Preliminary results of petrographic and electron-spin-resonance studies of organic matter from Deep Sea Drilling Project Sites 370 and 416. In Lancelot, Y., Winterer, E. L., et al., Init. Repts. DSDP, 50: Washington (U.S. Govt. Printing Office), 567-573.

Espitalié, J., Laporte, J. L., Madec, M., Marquis, F., Leplat, P., Paulet, J., and Boutefeu, A., 1977. Méthode rapide de caractérisation des roches mères, de leur potentiel pétrolier et de leur degré d'évolution. Rev. Inst. Fr. Pet., 32:23-42.

Hunt, J. M., Miller, R. J., and Whelan, J. K., 1980a. Formation of $\mathrm{C}_{4}-\mathrm{C}_{7}$ hydrocarbons from bacterial degradation of naturally occurring terpenoids. Nature (London), 288:577-578.

Hunt, J. M., Whelan, J. K., and Huc, A. Y., 1980b. Genesis of petroleum hydrocarbons in marine sediments. Science, 209:403-404.

Leythaeuser, D., Schaefer, R. G., Cornford, C., and Weiner, B., 1979. Generation and migration of light hydrocarbons $\left(\mathrm{C}_{2}-\mathrm{C}_{7}\right)$ in sedimentary basins. Org. Geochem., 1:191-204.

Leythaeuser, D., Schaefer, R. G., and Pooch, H., 1983. Diffusion of light hydrocarbons in subsurface sedimentary rocks. Am. Assoc. Pet. Geol. Bull., 67:889-895. 
Leythaeuser, D., Schaefer, R. G., and Yükler, A., 1980. Diffusion of light hydrocarbons through near-surface rocks. Nature (London), 284:522-525.

1982. Role of diffusion in primary migration of hydrocarbons. Am. Assoc. Pet. Geol. Bull., 66:408-429.

McAuliffe, C., 1966. Solubility in water of paraffin, cycloparaffin, olefin, acetylene, cycloolefin and aromatic hydrocarbons. J. Phys. Chem., 70:1267-1275.

Philippi, G. T., 1975. The deep subsurface temperature controlled origin of the gaseous and gasoline-range hydrocarbons of petroleum. Geochim. Cosmochim. Acta, 39:1353-1373.

Schaefer, R. G. and Leythaeuser, D., 1984. $\mathrm{C}_{2}-\mathrm{C}_{8}$ Hydrocarbons in sediments from Deep Sea Drilling Project Leg 75, Hole 530A (Angola Basin) and Hole 532 (Walvis Ridge), South Atlantic. In Hay, W. W., Sibuet, J.-C., et al., Init. Repts. DSDP, 75: Washington (U.S. Govt. Printing Office).

Schaefer, R. G., Leythaeuser, D., and von der Dick, H., 1983. Generation and migration of low-molecular-weight hydrocarbons in sediments from Site 511 of DSDP/IPOD Leg 71, Falkland Plateau, South Atlantic. In Bjorøy, M., et al. (Ed.), Advances in Organic Geochemistry 1981: Chichester (Wiley), pp. 164-174.

Schaefer, R. G., von der Dick, H., and Leythaeuser, D., 1983. $C_{2}-C_{8}$ Hydrocarbons in sediments from Deep Sea Drilling Project Leg 71, Site 511, Falkland Plateau, South Atlantic. In Ludwig, W. J., Krasheninnikov, V., et al., Init. Repts. DSDP, 71: Washington (U.S. Govt. Printing Office), 1033-1043.

Schaefer, R. G., Weiner, B., and Leythaeuser, D., 1978. Determination of sub-nanogram per gram quantities of light hydrocarbons $\left(\mathrm{C}_{2}-\mathrm{C}_{9}\right)$ in rock samples by hydrogen stripping in the flow system of a capillary gas chromatograph. Anal. Chem., 50:1848-1854.

Schaefer, R. G., Welte, D., and Pooch, H., in press. Geochemistry of low-molecular-weight hydrocarbons in two exploration wells of the
Elmworth gas field (Western Canada Basin). In Schenck, P., et al. (Eds.), Advances in Organic Geochemistry 1983: Oxford (Pergamon Press).

Thompson, K. F. M., 1979. Light hydrocarbons in subsurface sediments. Geochim. Cosmochim. Acta, 43:657-672.

Tissot, B., Califet-Debyser, Y., Deroo, G., and Oudin, J. L., 1971. Origin and evolution of hydrocarbons in early Toarcian shales, Paris Basin, France. Am. Assoc. Petrol. Geol. Bull., 55:2177-2193.

Whelan, J. K., 1979. $C_{1}$ to $C_{7}$ Hydrocarbons from IPOD Holes 397 and 397A. In von Rad, U., Ryan, W. B. F., et al., Init. Repts. DSDP , 47, Pt. 1: Washington (U.S. Govt. Printing Office), 531-539.

Whelan, J. K., and Hunt, J. M., 1980. Sediment $C_{1}$ to $C_{7}$ Hydrocarbons from Deep Sea Drilling Project Sites 415 and 416 (Moroccon Basin). In Lancelot, Y., Winterer, E. L., et al., Init. Repts. DSDP, 50: Washington (U.S. Govt. Printing Office), 623-624.

1981. $C_{1}-C_{8}$ Hydrocarbons in Leg 63 sediments from outer California and Baja California borderlands. In Yeats, R. S., Haq, B. U., et al., Init. Repts. DSDP, 63: Washington (U.S. Govt. Printing Office), 775-784.

1982. $C_{1}-C_{8}$ Hydrocarbons in Leg 64 sediments, Gulf of California. In Curray, J. R., Moore, D. G., et al., Init. Repts. $D S D P, 64$, Pt. 2: Washington (U.S. Govt. Printing Office), 763-780.

Whelan, J. K., Hunt, J. M., and Berman, J., 1980. Volatile $C_{1}-C_{7}$ organic compounds in surface sediments from Walvis Bay. Geochim. Cosmochim. Acta, 44:1767-1785.

Whelan, J. K., Tarafa, M. E., and Hunt, J. M., 1982. Volatile $C_{1}-C_{8}$ organic compounds in macroalgae. Nature (London), 299:50-52.

Date of Initial Receipt: June 10, 1983

Date of Acceptance: January 3, 1984 


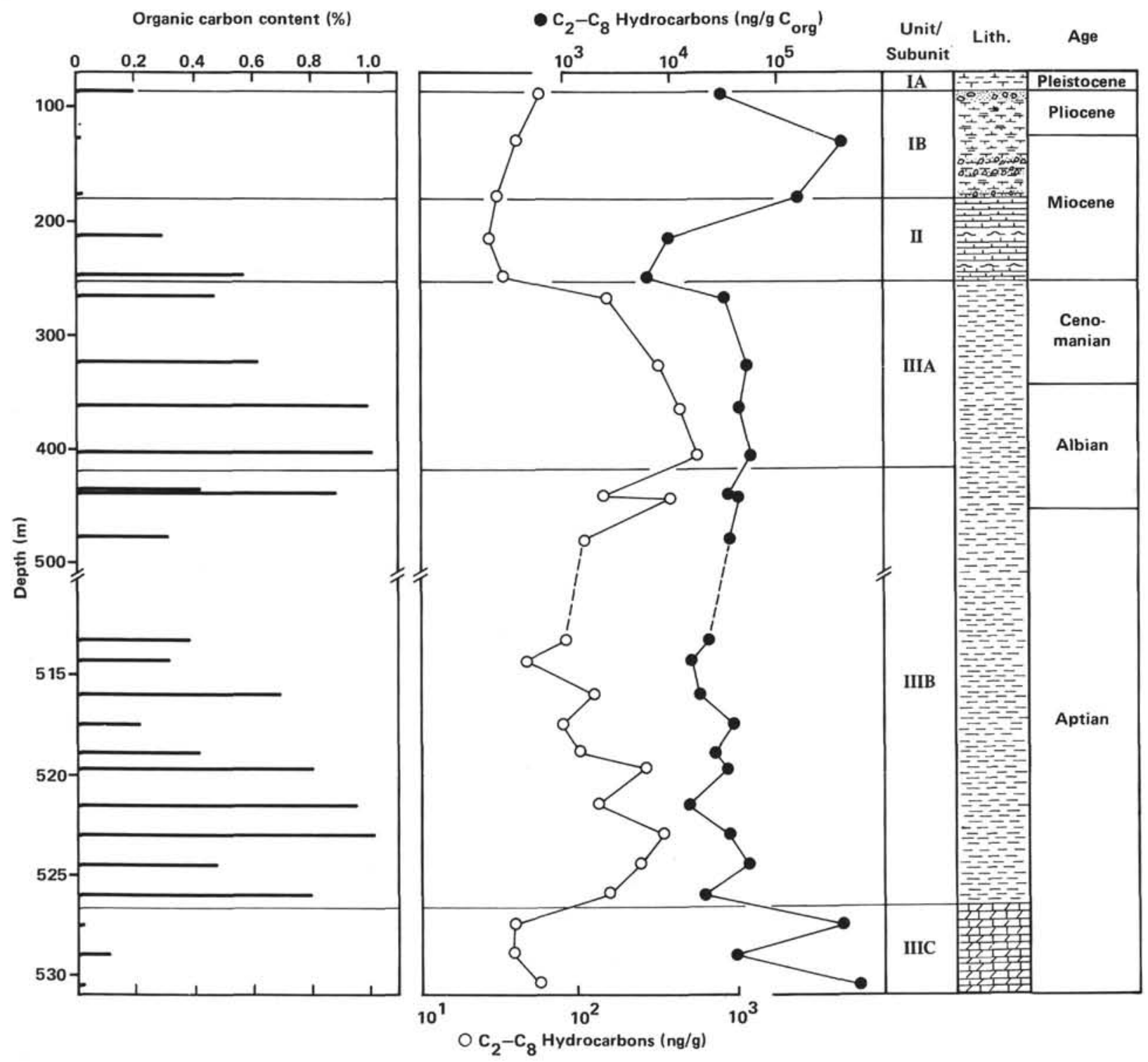

Figure 1. Organic carbon and low-molecular-weight hydrocarbon content (sum of all $\mathrm{C}_{2}-\mathrm{C}_{8}$ hydrocarbons identified in Table 1, except olefins) vs. depth for sediment samples from DSDP Site 545, Leg 79. Note change in depth scale. Lithologic units (schematic) and stratigraphy as defined by Leg 79 shipboard scientists. 


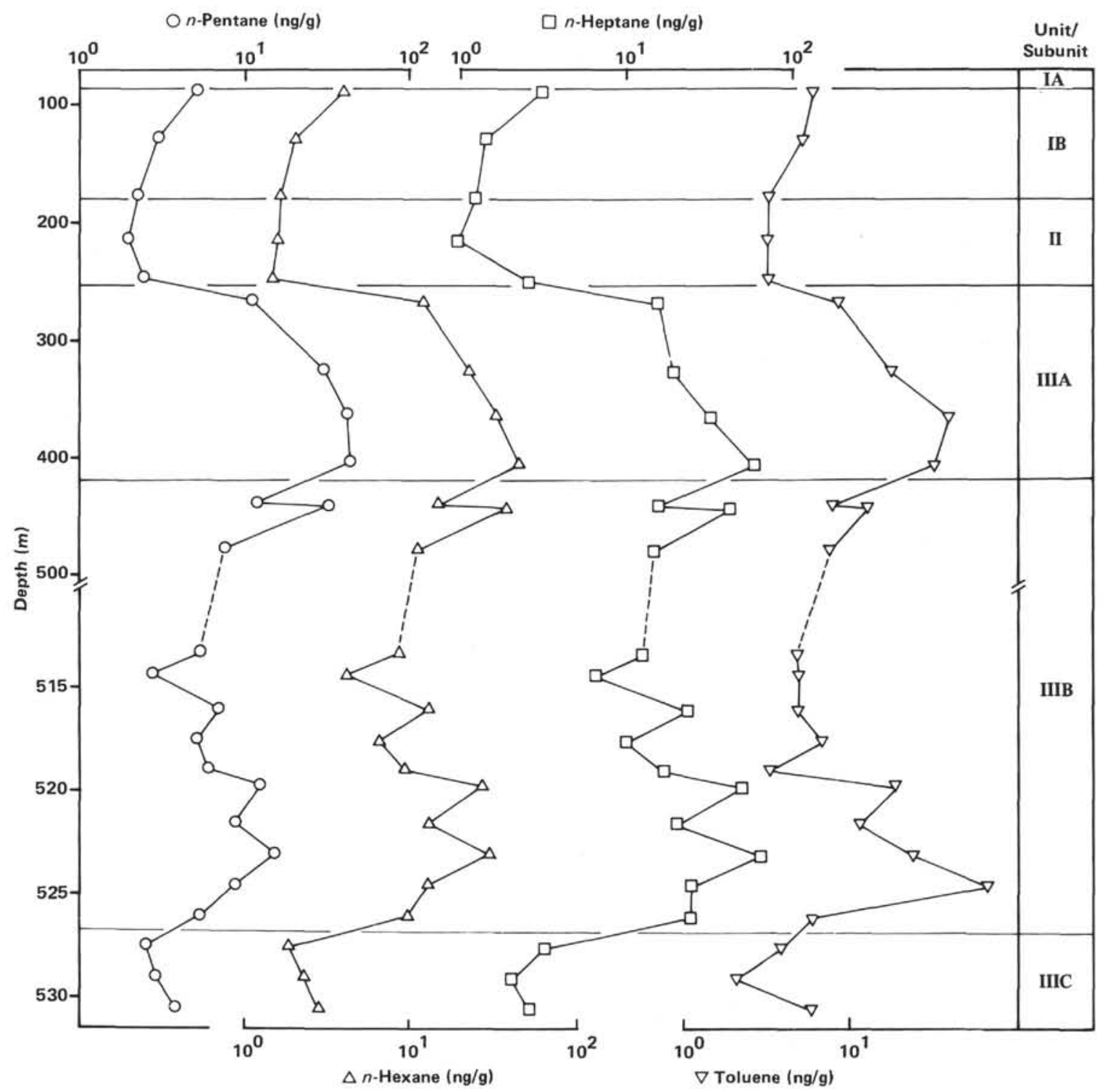

Figure 2. Ethane, propane, and $n$-butane concentrations (in $\mathrm{ng} / \mathrm{g}$ of dry sediment) versus depth for sediment samples from DSDP Site 545, Leg 79. 


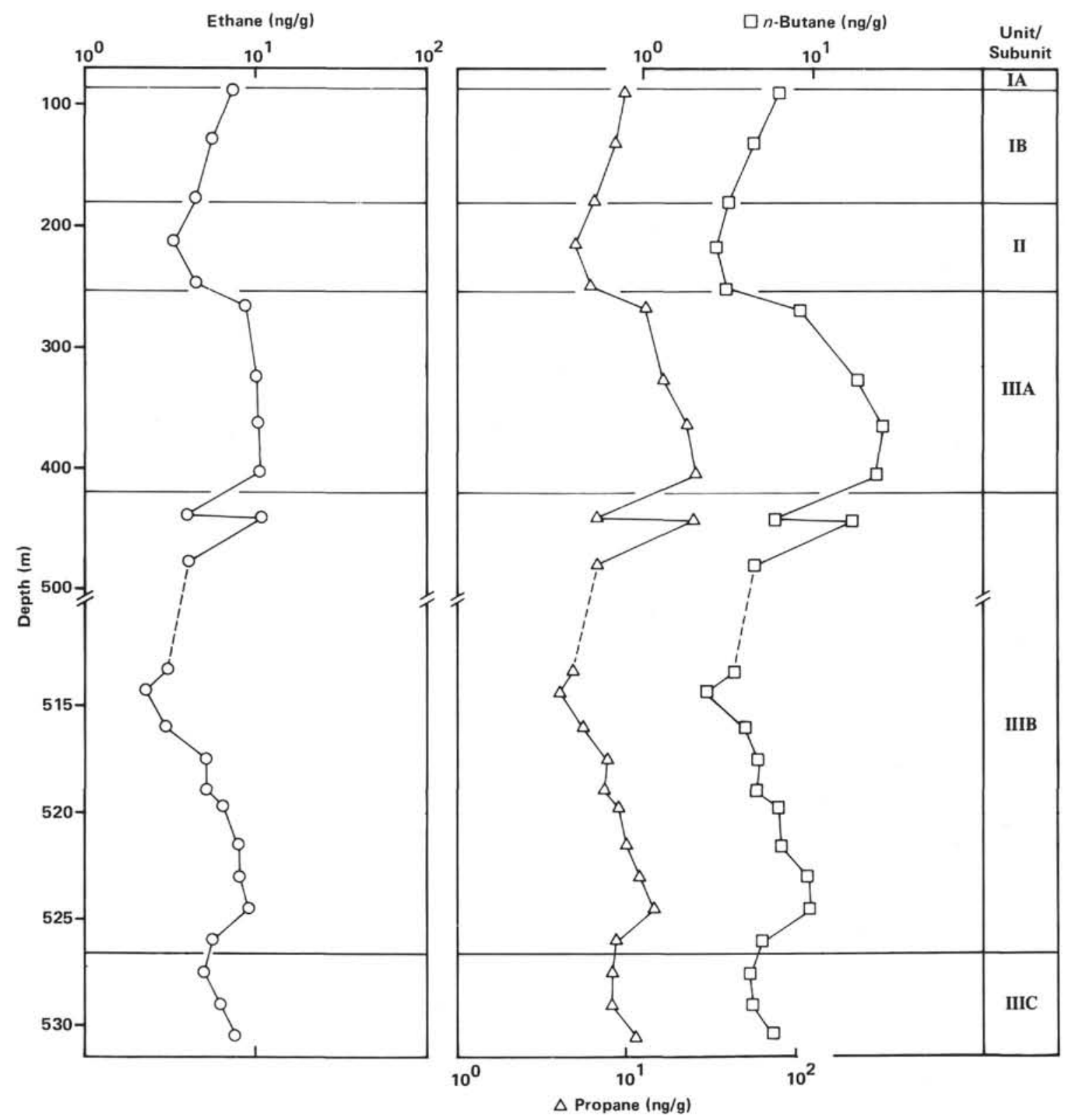

Figure 3. $n$-Pentane, $n$-hexane, $n$-heptane, and toluene concentrations (in ng/g of dry sediment) versus depth for sediment samples from DSDP Site 545, Leg 79. 
R. G. SCHAEFER, D. LEYTHAEUSER, J. GORMLY

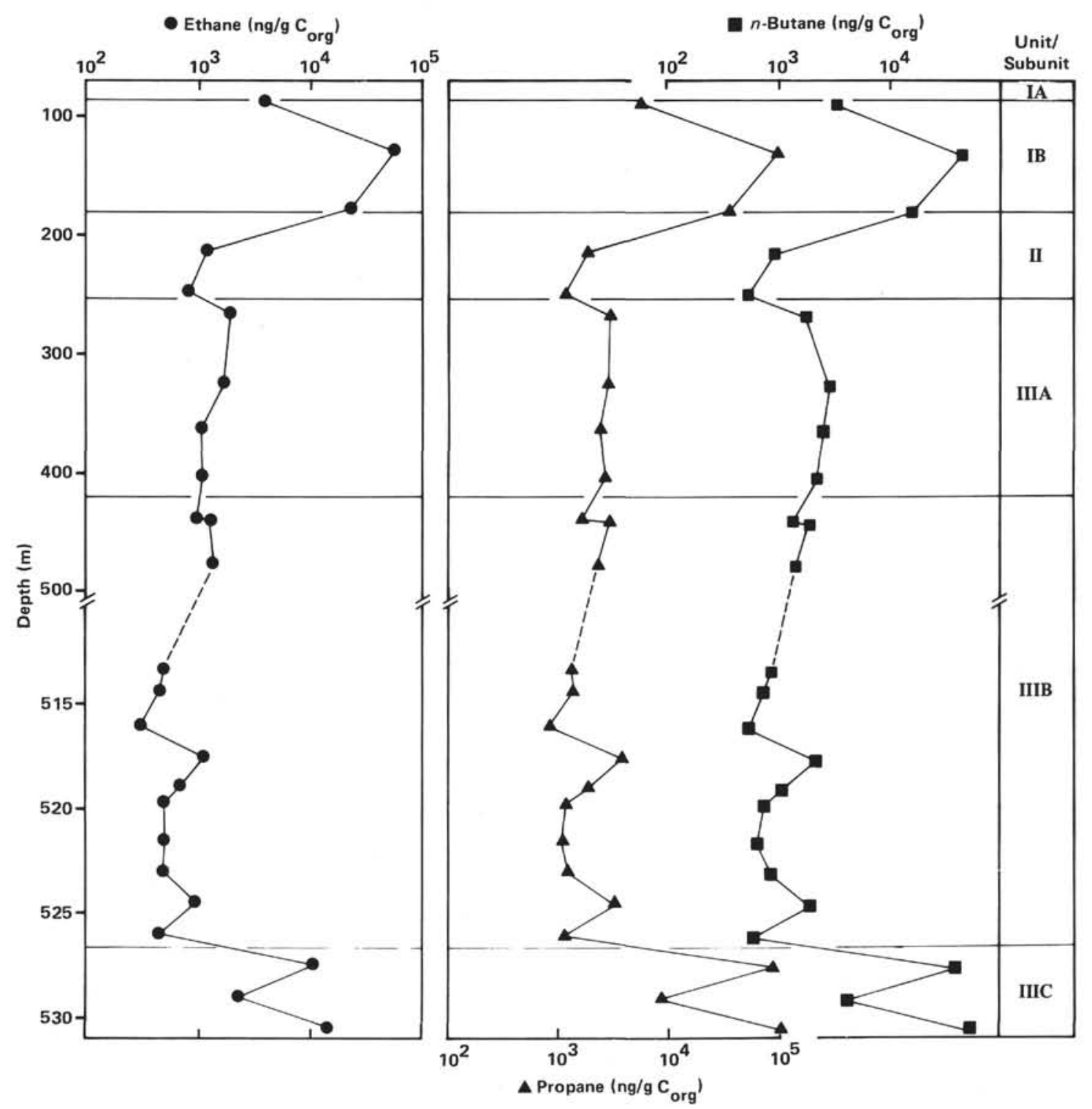

Figure 4. Organic-carbon-normalized ethane, propane, and $n$-butane concentrations (in $\mathrm{ng} / \mathrm{g} \mathrm{C}_{\text {org }}$ ) versus depth for sediment samples from DSDP Site 545, Leg 79. 


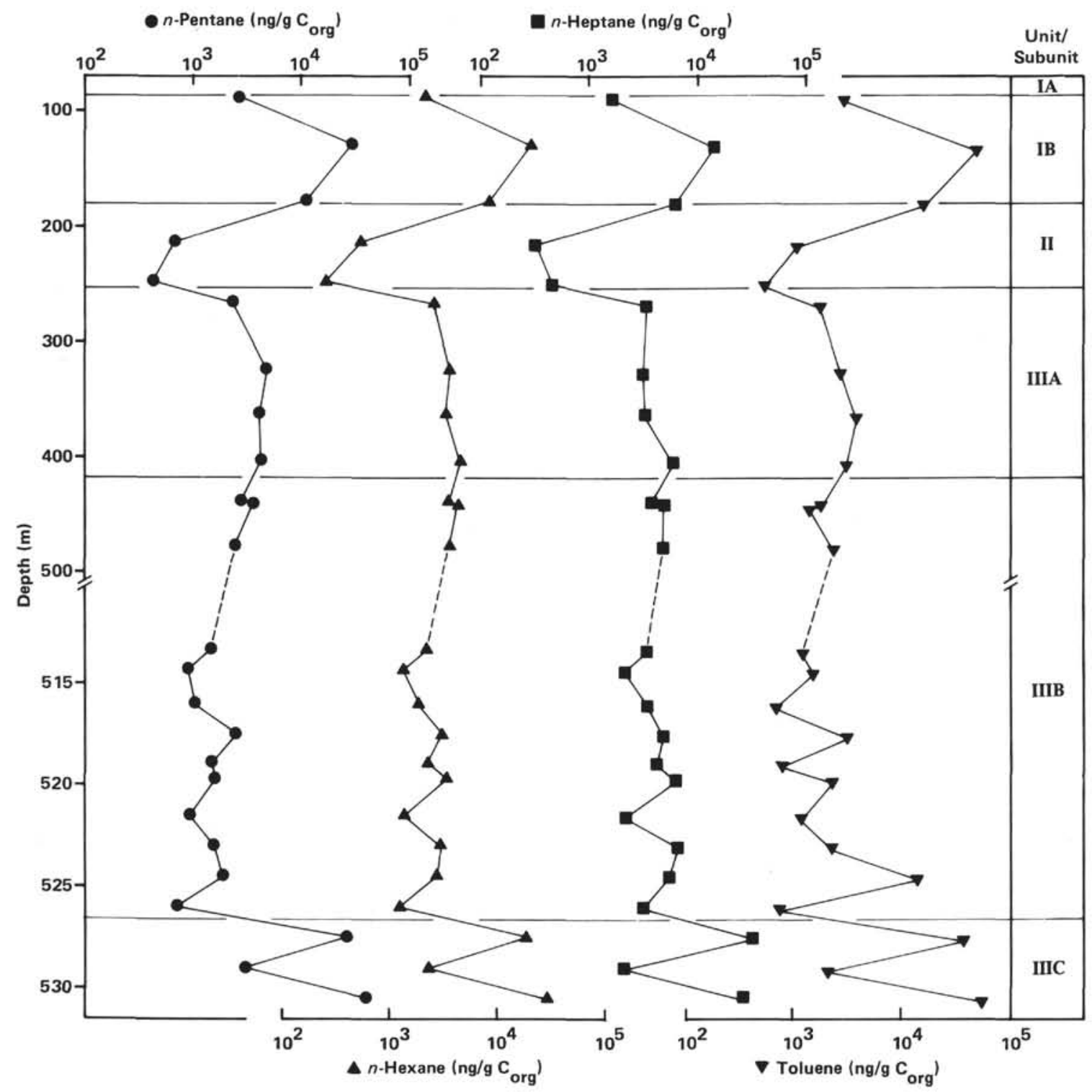

Figure 5. Organic-carbon-normalized $n$-pentane, $n$-hexane, $n$-heptane, and toluene concentrations (in ng/g $\mathrm{C}_{\text {org }}$ ) versus depth for sediment samples from DSDP Site 545, Leg 79. 


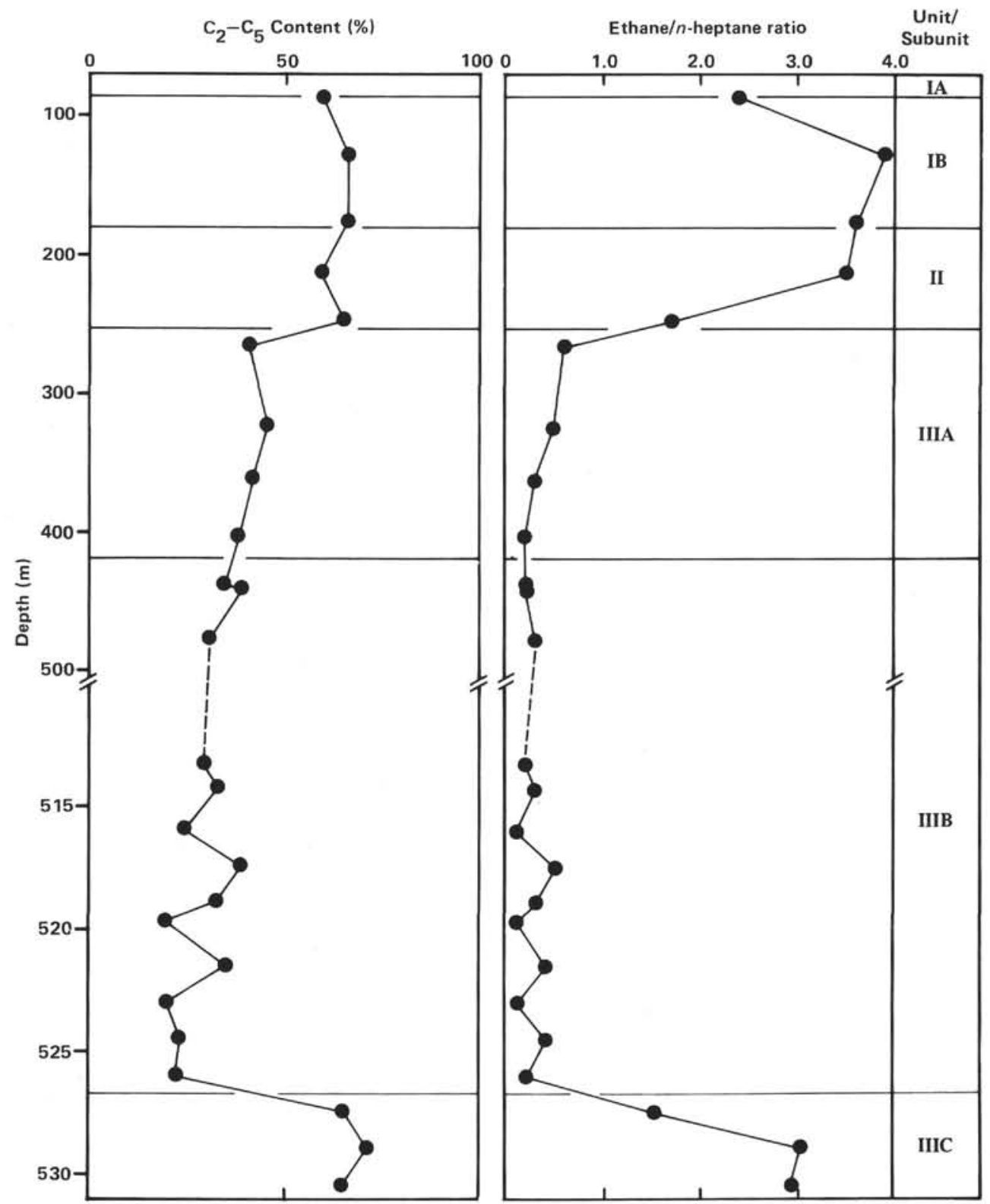

Figure 6. $\mathrm{C}_{2}-\mathrm{C}_{5}$ Hydrocarbon content (as a proportion of total $\mathrm{C}_{2}-\mathrm{C}_{8}$ hydrocarbons) and ethane/ $n$-heptane concentration ratio vs. depth for sediment samples from DSDP Site 545, Leg 79. 


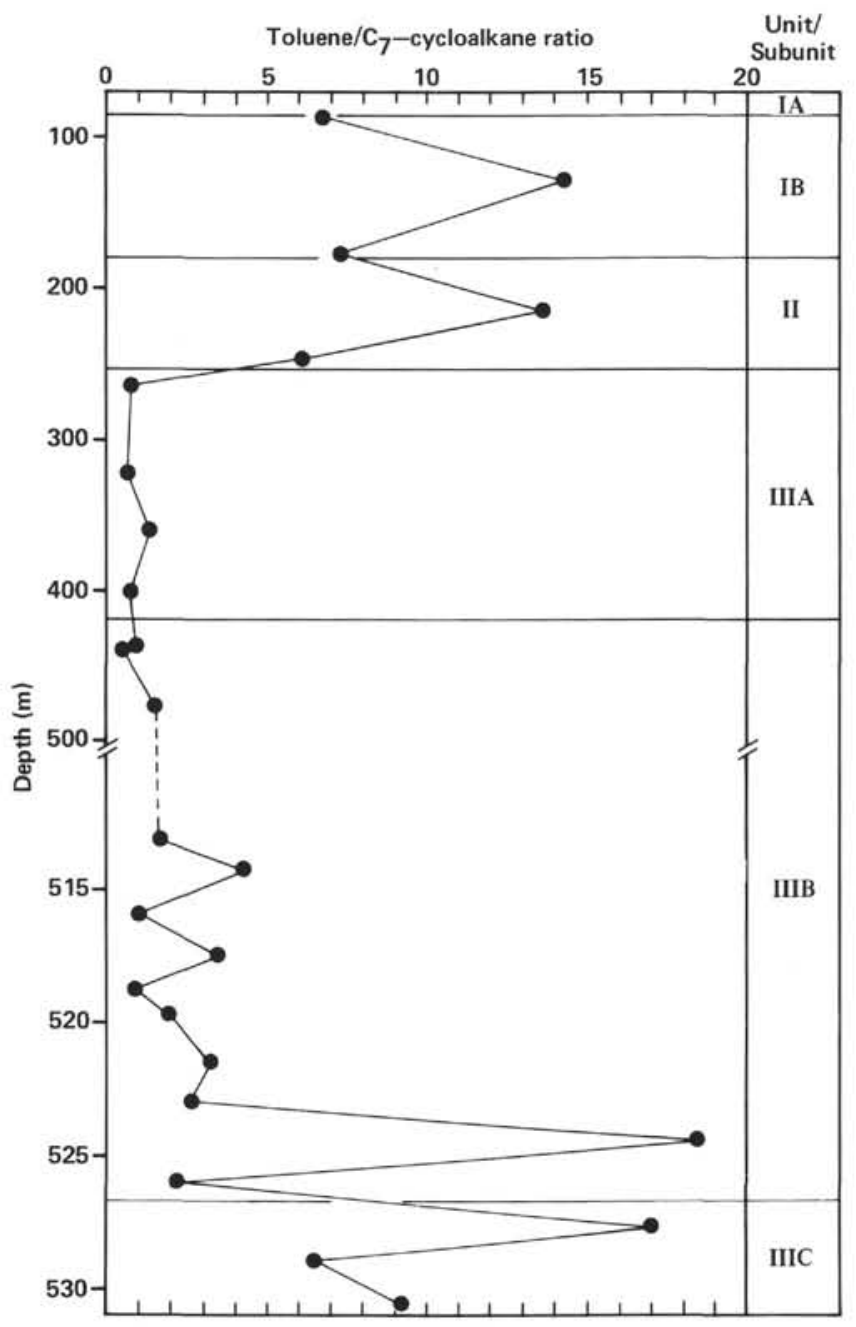

Figure 7. Toluene/ $\mathrm{C}_{7}$-cycloalkane (sum of compounds $26,27,28,30$, 31 , and 33 in Table 1) concentration ratio versus depth for sediment samples from DSDP Site 545, Leg 79. 


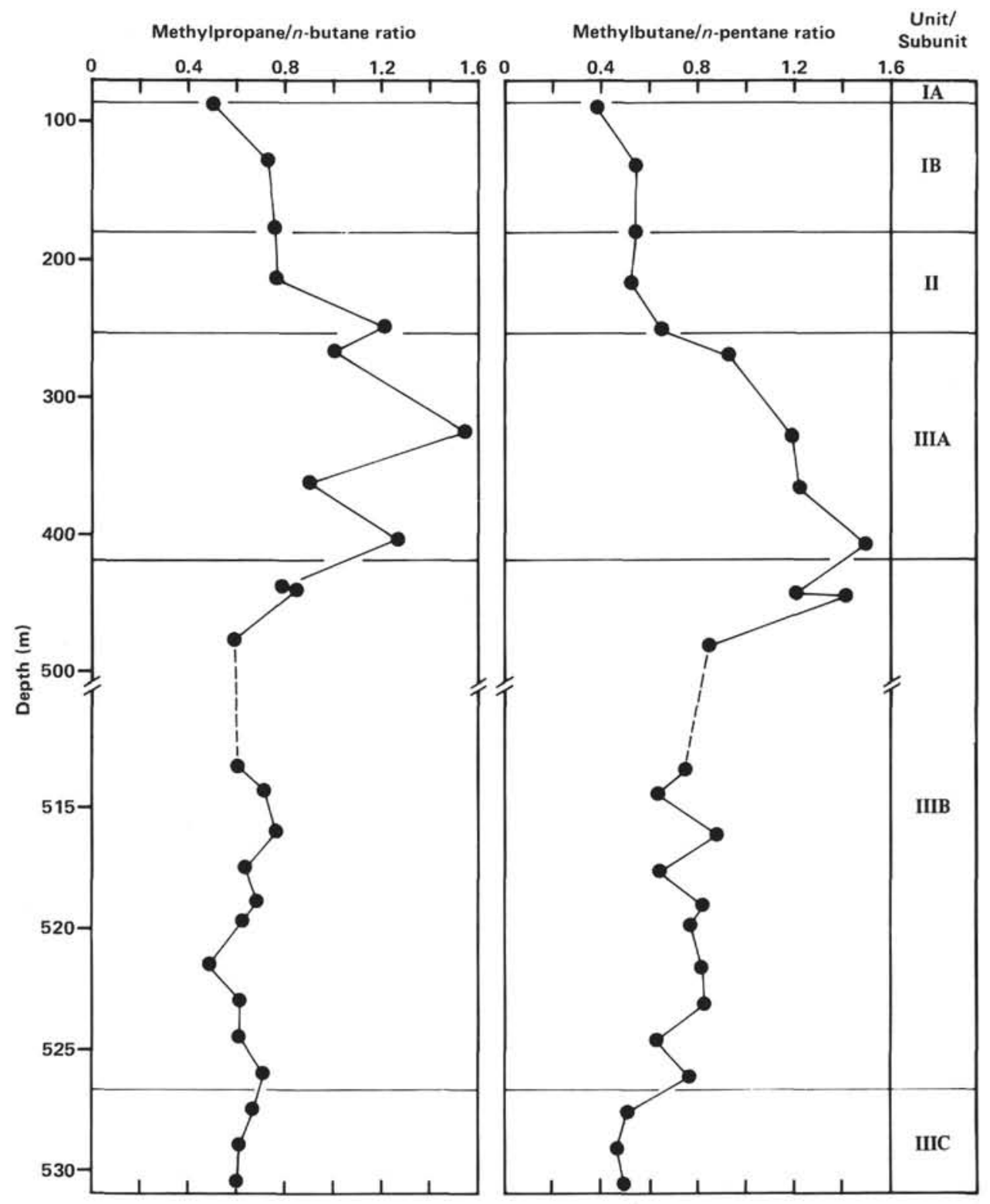

Figure 8. Methylpropane/ $n$-butane and methylbutane/ $n$-pentane concentration ratios versus depth for sediment samples from DSDP Site 545, Leg 79. 


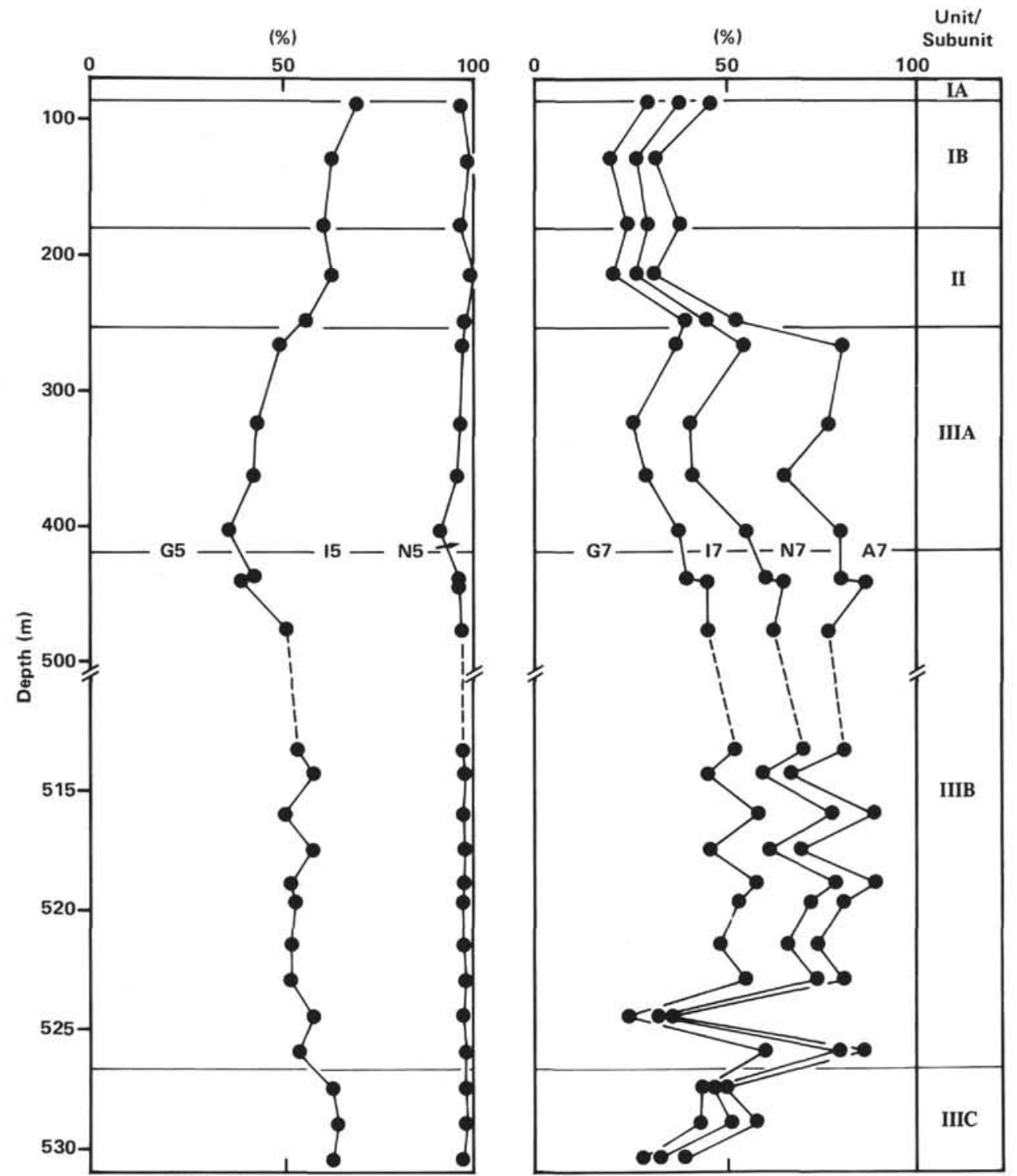

Figure 9. Relative composition of $\mathrm{C}_{5}$ and $\mathrm{C}_{7}$ hydrocarbons versus depth for sediment samples from DSDP Site 545, Leg 79. G5 = $n$-pentane, I5 = $\mathrm{C}_{5}$ branched alkanes; N5 = cyclopentane (sum equals $100 \%$, plotted from left to right); $\mathrm{G} 7=n$-heptane, $\mathrm{I} 7=\mathrm{C}_{7}$ branched alkanes; $\mathrm{N} 7=\mathrm{C}_{7}$ cycloalkanes, $\mathrm{A} 7=$ toluene (sum equals $100 \%$, plotted from left to right). 

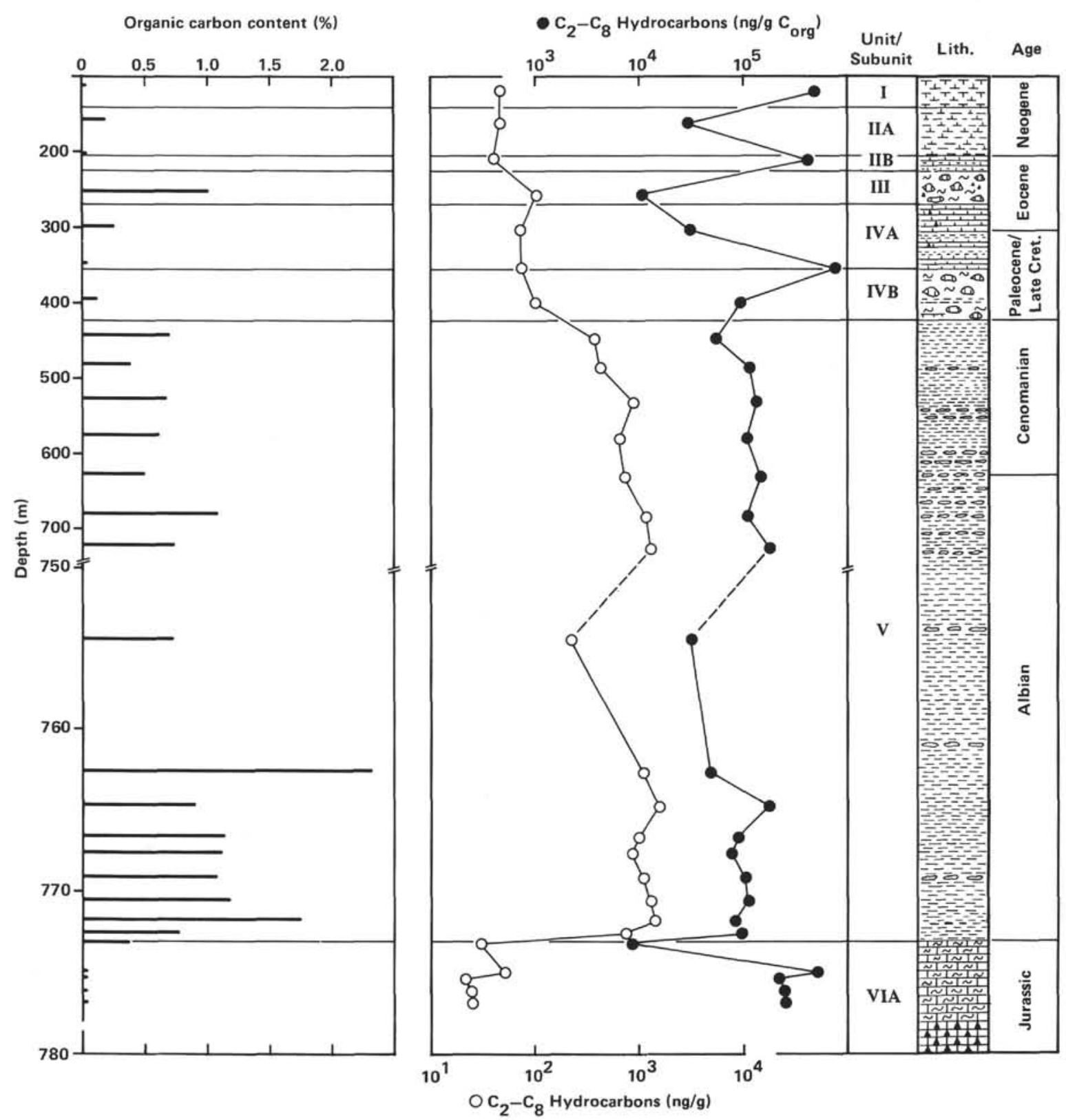

Figure 10. Organic-carbon and low-molecular weight hydrocarbon content (sum of all $\mathrm{C}_{2}-\mathrm{C}_{8}$ hydrocarbons identified in Table 1, except olefins) vs. depth for sediment samples from DSDP Holes 547A and 547B, Leg 79. Note change in depth scale. Lithologic units (schematic) and stratigraphy as defined by Leg 79 shipboard scientists. 


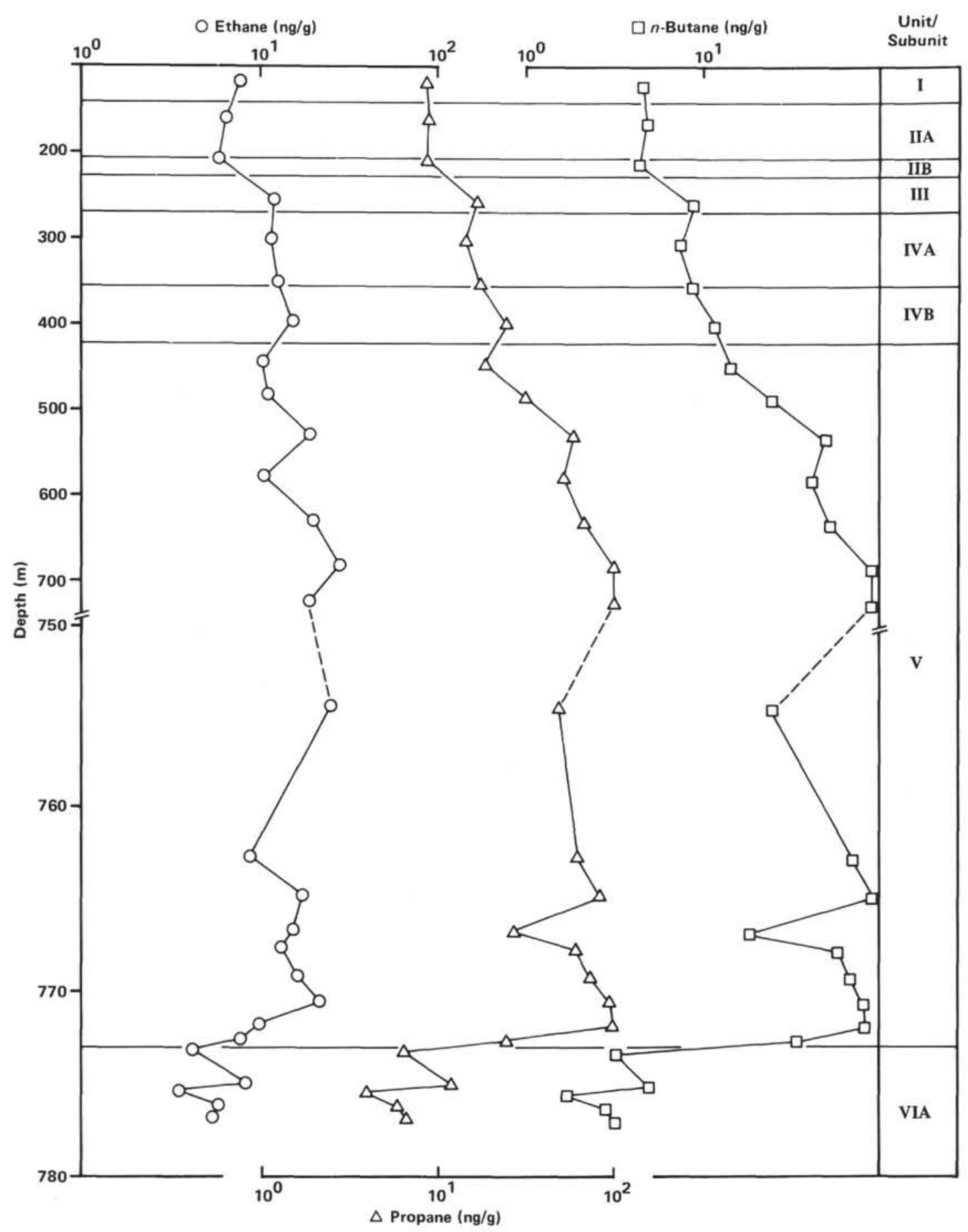

Figure 11. Ethane, propane, and $n$-butane concentrations (in $\mathrm{ng} / \mathrm{g}$ of dry sediment) versus depth for sediment samples from DSDP Holes 547A and 547B, Leg 79. 


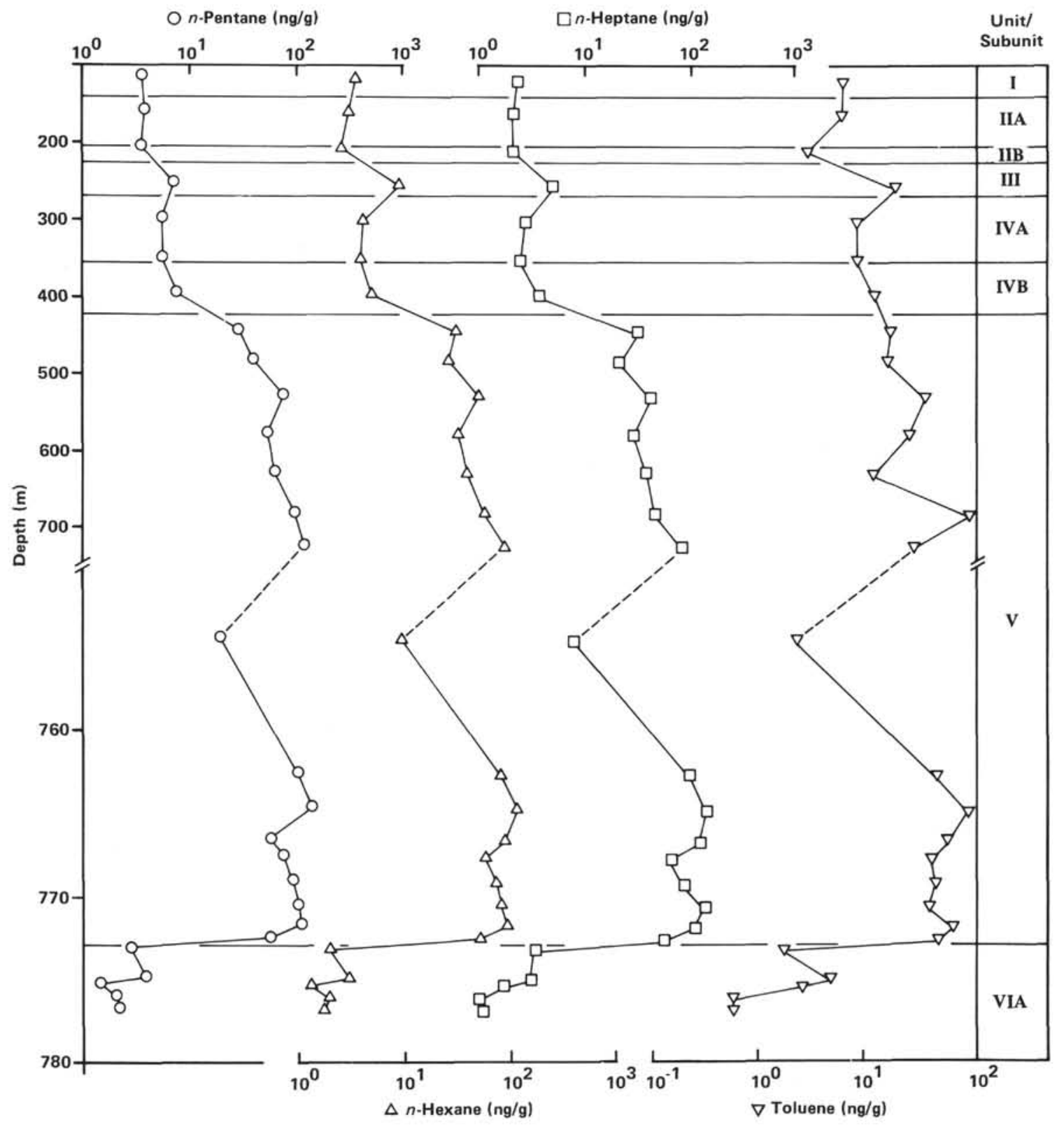

Figure 12. $n$-Pentane, $n$-hexane, $n$-heptane, and toluene concentrations (in $\mathrm{ng} / \mathrm{g}$ of dry sediment) versus depth for sediment samples from DSDP Holes 547A and 547B, Leg 79. 


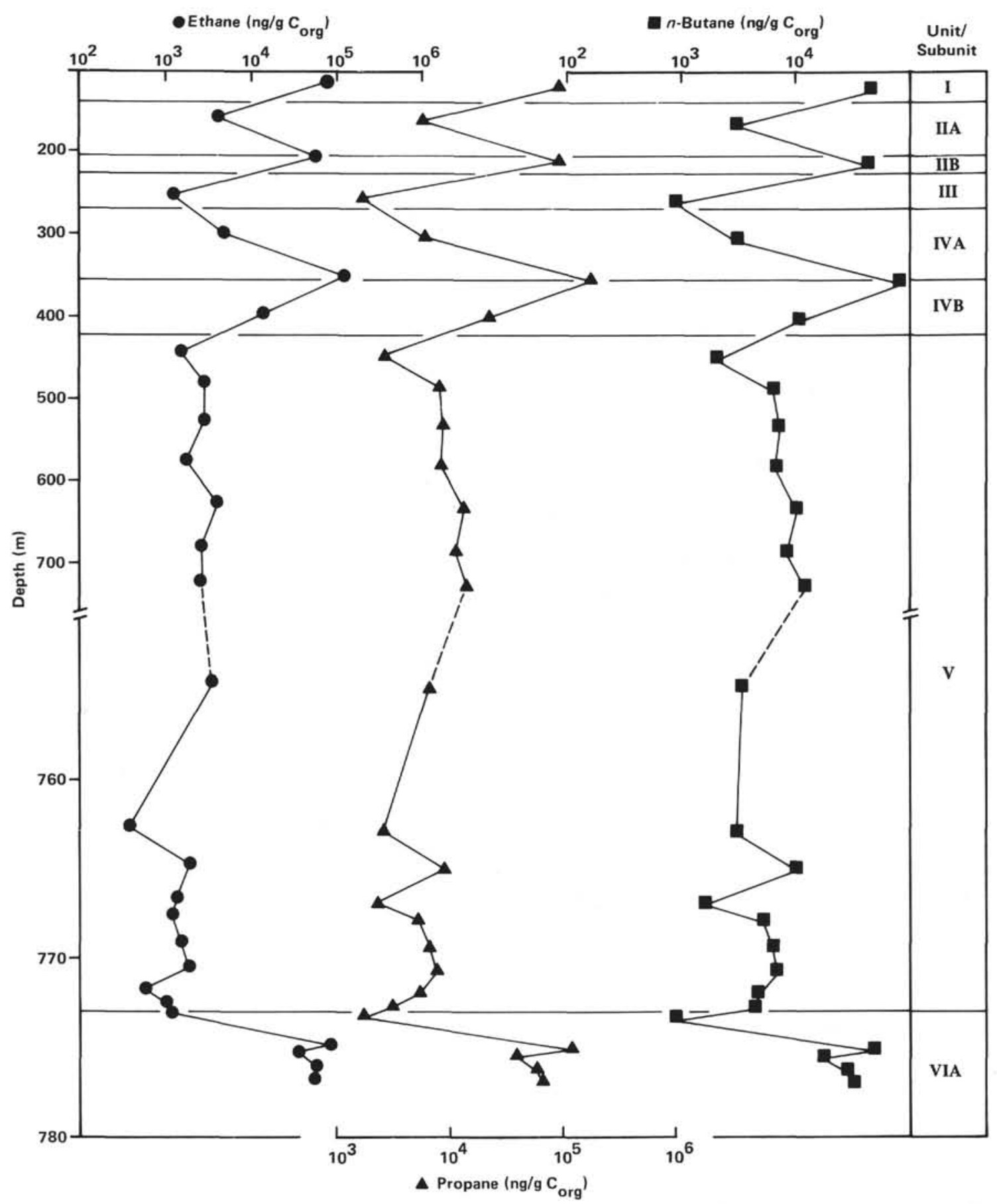

Figure 13. Organic-carbon normalized ethane, propane, and $n$-butane concentrations (in $\mathrm{ng} / \mathrm{g} \mathrm{C}_{\text {org }}$ ) versus depth for sediment samples from DSDP Holes 547A and 547B, Leg 79. 


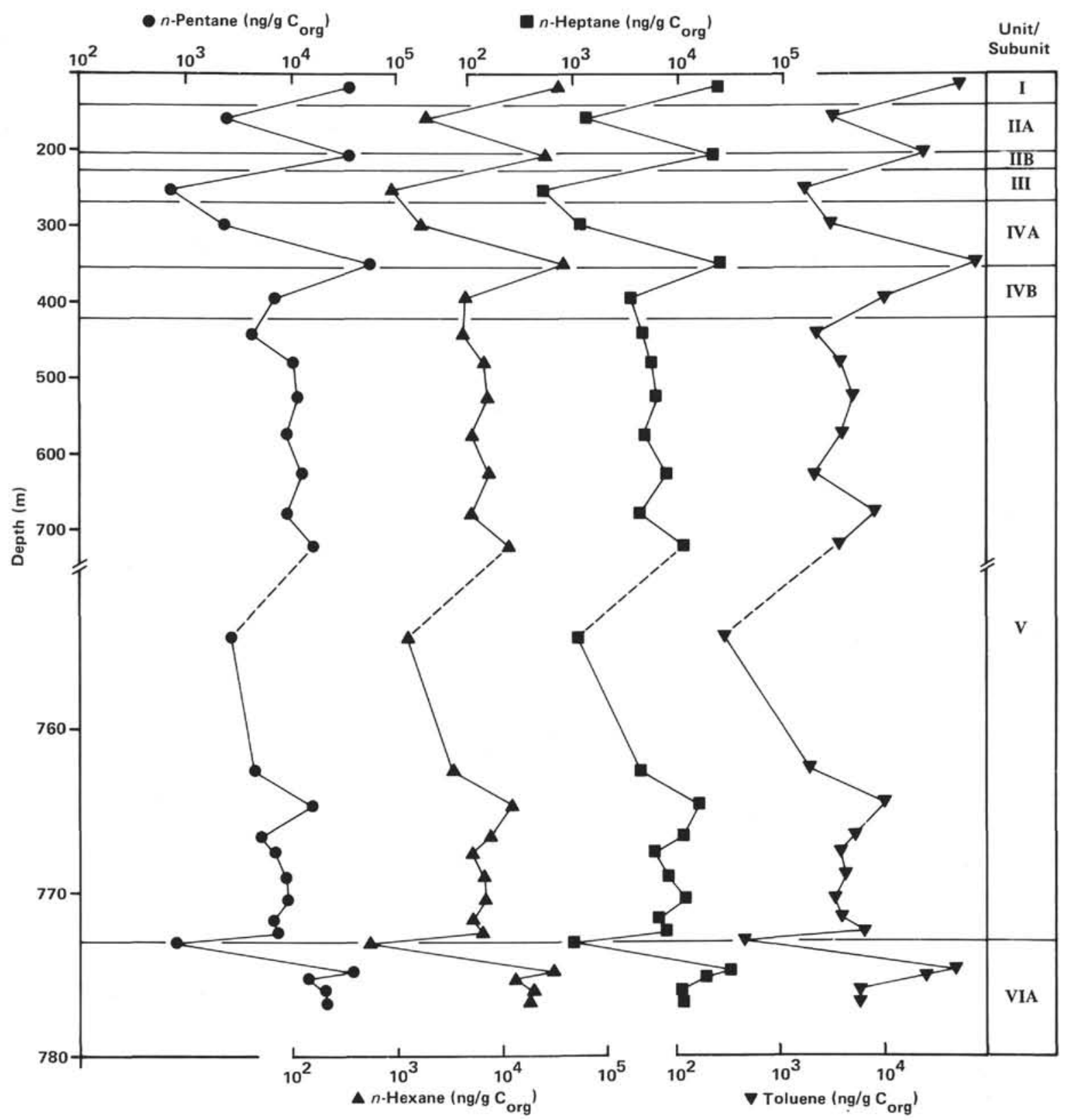

Figure 14. Organic-carbon normalized $n$-pentane, $n$-hexane, $n$-heptane, and toluene concentrations (in ng/g $\mathrm{C}_{\text {org }}$ ) versus depth for sediment samples from DSDP Holes 547A and 547B, Leg 79. 


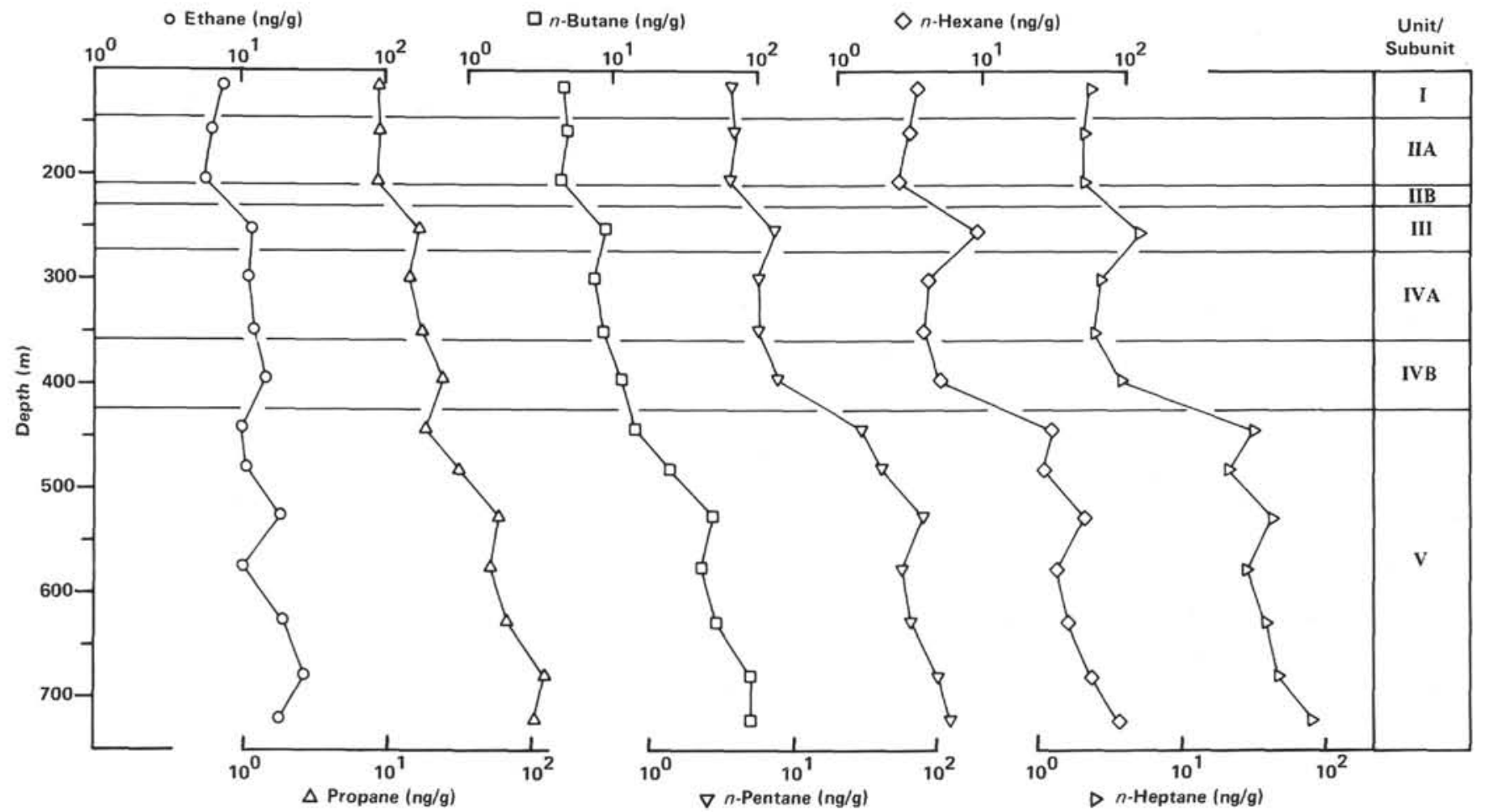

Figure 15. $n$-Alkane concentrations (ethane through $n$-heptane) versus depth for sediment samples of DSDP Hole 547A, Leg 79.

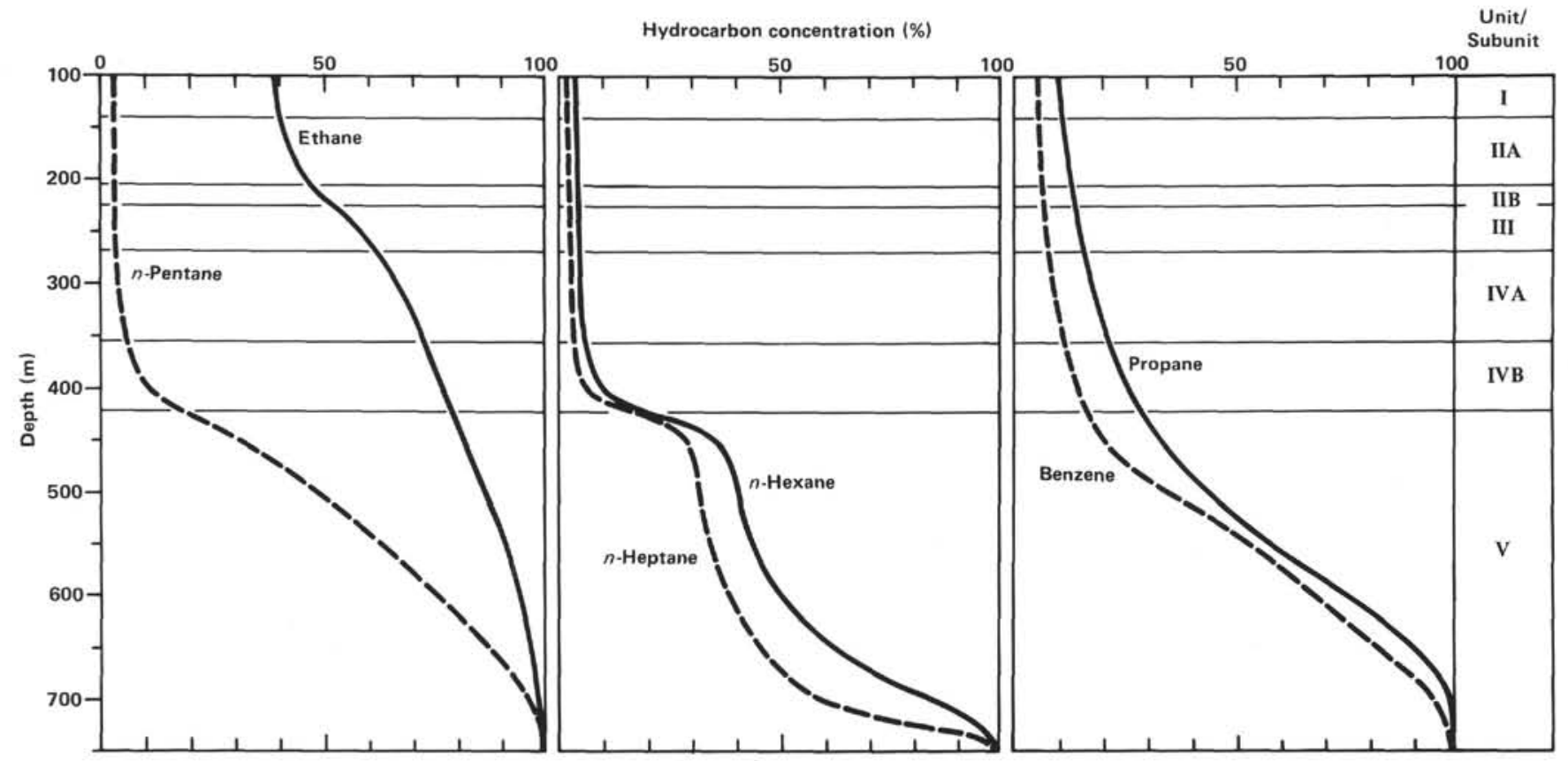

Figure 16. Relative change of selected light hydrocarbon concentrations versus depth for sediment samples of DSDP Hole 547A, Leg 79. 


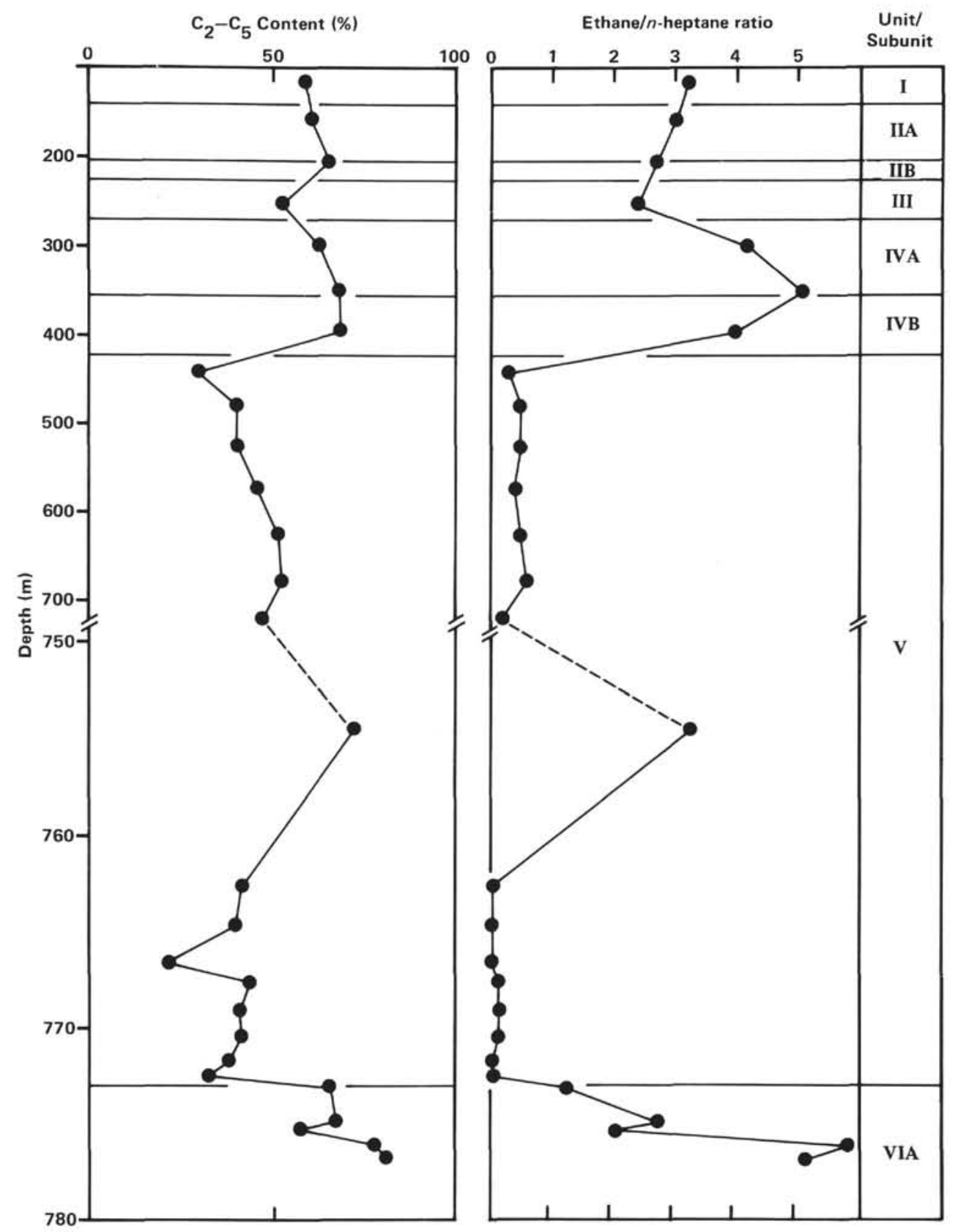

Figure 17. $C_{2}-C_{5}$ Hydrocarbon content (as a proportion of total $C_{2}-C_{8}$ hydrocarbons) and ethane/ $n$-heptane concentration ratio versus depth for sediment samples from DSDP Holes 547A and 547B, Leg 79. 


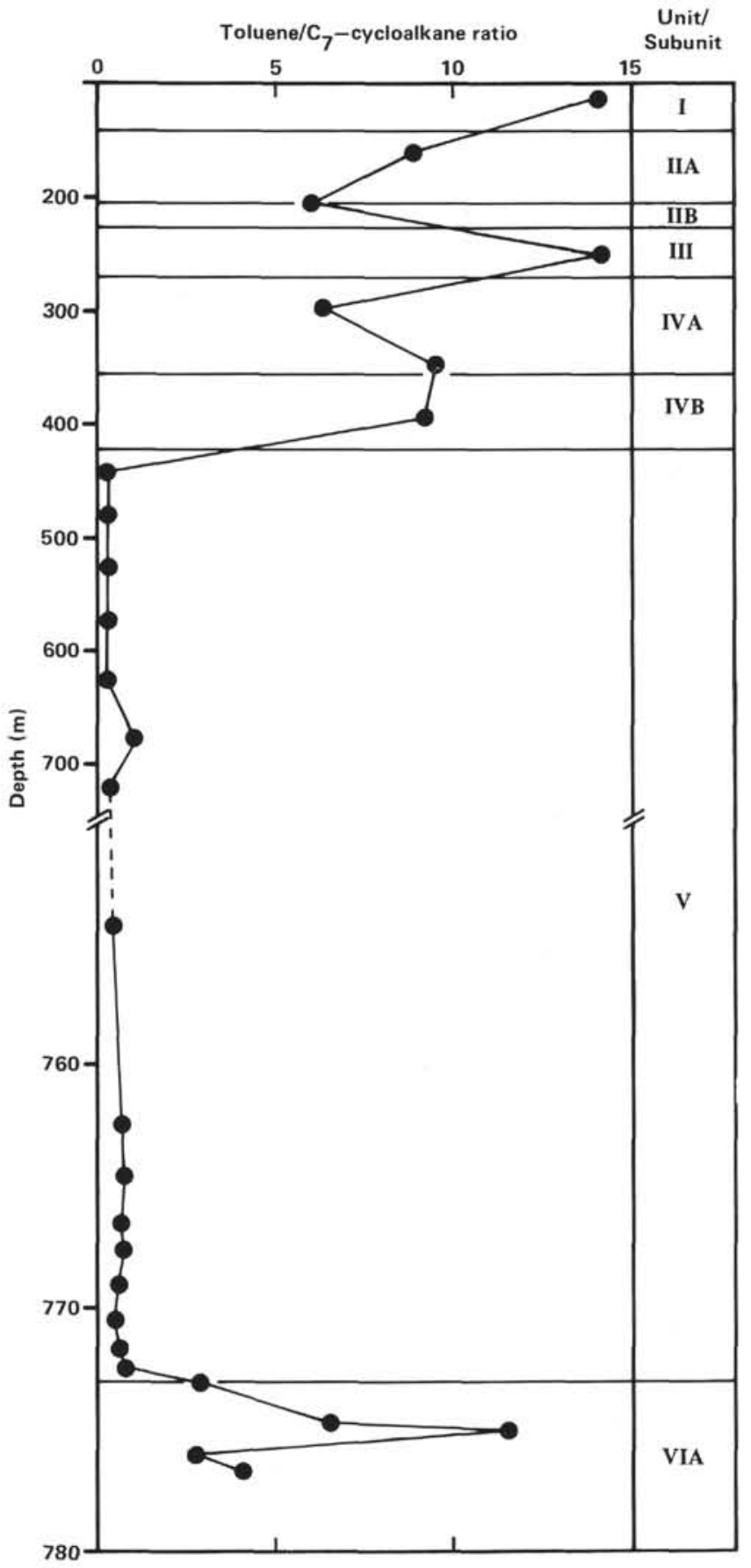

Figure 18. Toluene $/ C_{7}$-cycloalkane (sum of compounds $26,27,28,30$, 31 , and 33 in Table 1) concentration ratio versus depth for sediment samples from DSDP Holes 547A and 547B, Leg 79. 


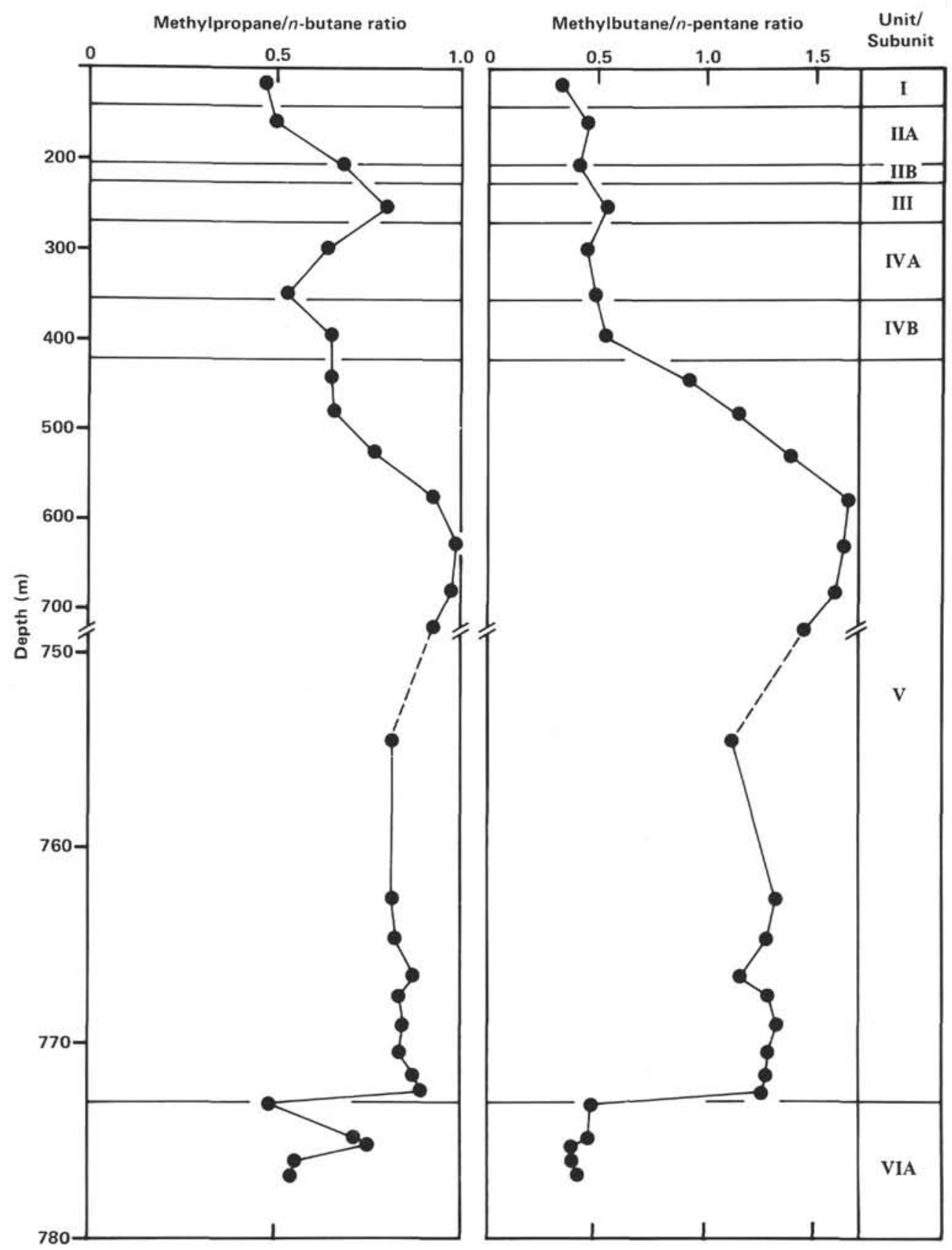

Figure 19. Methylpropane/ $n$-butane and methylbutane/n-pentane concentration ratios versus depth for sediment samples from DSDP Holes 547A and 547B, Leg 79. 


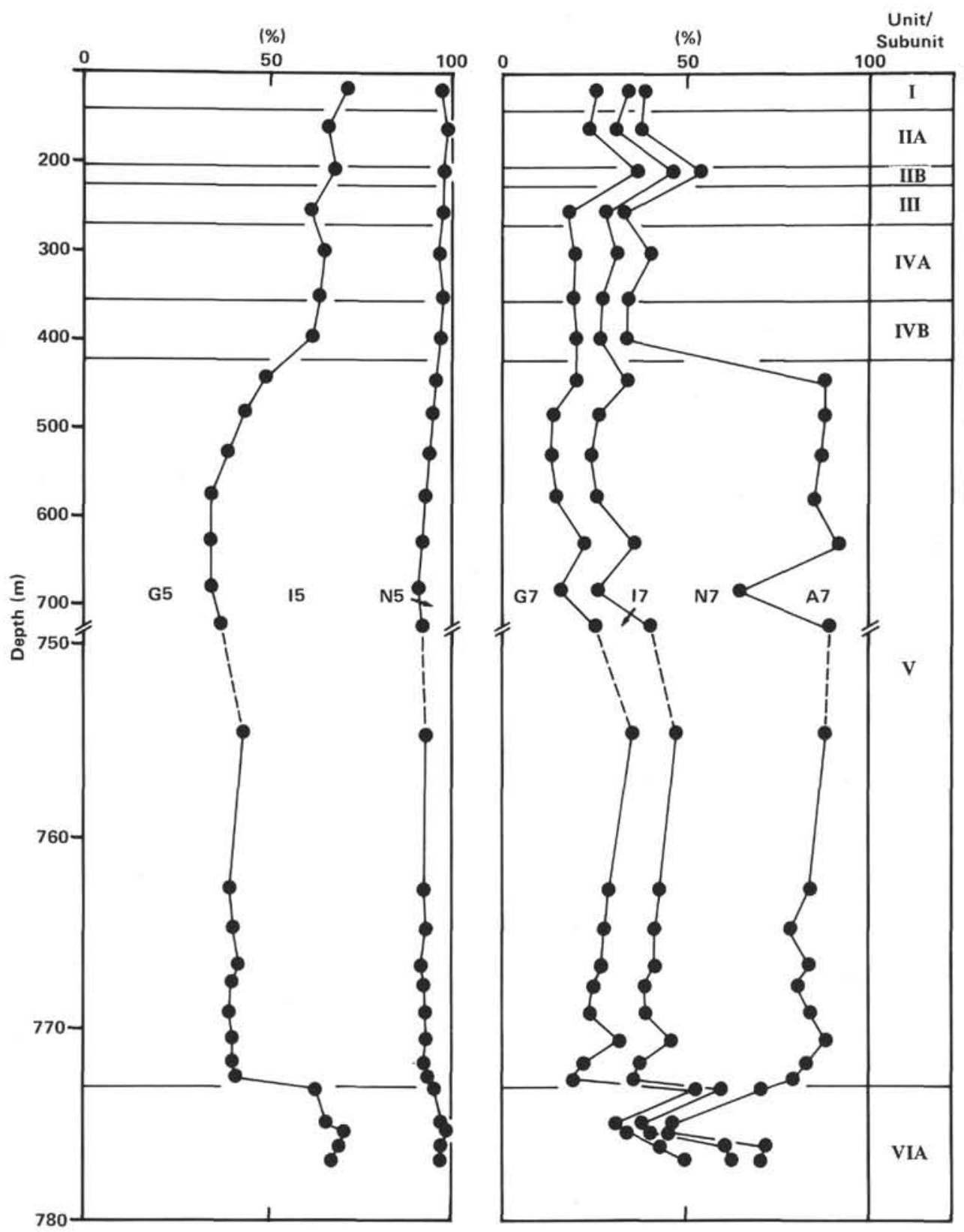

Figure 20. Relative composition of $\mathrm{C}_{5}$ and $\mathrm{C}_{7}$ hydrocarbons versus depth for sediment samples from DSDP Holes 547A and 547B, Leg 79. G5 $=n$-pentane, I5 = $\mathrm{C}_{5}$ branched alkanes; N5 = cyclopentane (sum equals $100 \%$, plotted from left to right); $\mathrm{G} 7=n$-heptane, $\mathrm{I} 7=\mathrm{C}_{7}$ branched alkanes; $\mathrm{N} 7=$ $\mathrm{C}_{7}$ cycloalkanes, $\mathrm{A} 7=$ toluene (sum equals $100 \%$, plotted from left to right). 